STRUCTURAL SCIENCE CRYSTAL ENGINEERING MATERIALS

ISSN 2052-5206

Received 14 July 2020

Accepted 7 October 2020

Edited by J. Hadermann, University of Antwerp, Belgium

Keywords: perovskite; nanoparticle exsolution; catalysis; DFT.

CCDC references: 2035990; 2035991; 2035992; 2035993; 2035994; 2035995; $2035996 ; 2035997$

Supporting information: this article has supporting information at journals.iucr.org/b

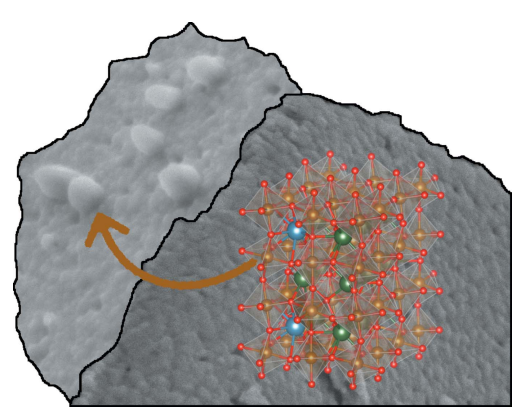

\section{Ca-doped rare earth perovskite materials for tailored exsolution of metal nanoparticles}

\author{
Lorenz Lindenthal, ${ }^{a}$ Thomas Ruh, ${ }^{a}$ Raffael Rameshan, ${ }^{a}$ Harald Summerer, ${ }^{\text {a,b }}$ \\ Andreas Nenning, ${ }^{b}$ Christopher Herzig, ${ }^{b}$ Stefan Löffler, ${ }^{c}$ Andreas Limbeck, \\ Alexander Karl Opitz, ${ }^{b}$ Peter Blaha $^{a}$ and Christoph Rameshan ${ }^{\mathrm{a} *}$
}

a Institute of Materials Chemistry, TU Wien, Getreidmarkt 9/165, Vienna 1060, Austria, ${ }^{\mathbf{b}}$ Institute of Chemical
Technologies and Analytics, TU Wien, Getreidmarkt 9/164, Vienna 1060, Austria, and ' ${ }^{\mathbf{C}}$ UTEM, TU Wien, Wiedner
Hauptstraße 8-10/E057-02, Vienna 1060, Austria. *Correspondence e-mail: christoph.rameshan@tuwien.ac.at

Perovskite-type oxide materials (nominal composition $A B \mathrm{O}_{3}$ ) are a very versatile class of materials, and their properties are tuneable by varying and doping $A$ - and $B$-site cations. When the $B$-site contains easily reducible cations (e.g. $\mathrm{Fe}, \mathrm{Co}$ or $\mathrm{Ni}$ ), these can exsolve under reducing conditions and form metallic nanoparticles on the surface. This process is very interesting as a novel route for the preparation of catalysts, since oxide surfaces decorated with finely dispersed catalytically active (often metallic) nanoparticles are a key requirement for excellent catalyst performance. Five doped perovskites, namely, $\mathrm{La}_{0.9} \mathrm{Ca}_{0.1} \mathrm{FeO}_{3-\delta}, \quad \mathrm{La}_{0.6} \mathrm{Ca}_{0.4} \mathrm{FeO}_{3-\delta}, \quad \mathrm{Nd}_{0.9} \mathrm{Ca}_{0.1} \mathrm{FeO}_{3-\delta}, \quad \mathrm{Nd}_{0.6} \mathrm{Ca}_{0.4} \mathrm{FeO}_{3-\delta}$ and $\mathrm{Nd}_{0.6} \mathrm{Ca}_{0.4} \mathrm{Fe}_{0.9} \mathrm{Co}_{0.1} \mathrm{O}_{3-\delta}$, have been synthesized and characterized by experimental and theoretical methods with respect to their crystal structures, electronic properties, morphology and exsolution behaviour. All are capable of exsolving Fe and/or Co. Special emphasis has been placed on the influence of the $A$-site elemental composition on structure and exsolution capability. Using $\mathrm{Nd}$ instead of La increased structural distortions and, at the same time, hindered exsolution. Increasing the amount of $\mathrm{Ca}$ doping also increased distortions and additionally changed the $\mathrm{Fe}$ oxidation states, resulting in exsolution being shifted to higher temperatures as well. Using the easily reducible element Co as the $B$-site dopant significantly facilitated the exsolution process and led to much smaller and homogeneously distributed exsolved particles. Therefore, the Codoped perovskite is a promising material for applications in catalysis, even more so as Co is catalytically a highly active element. The results show that fine-tuning of the perovskite composition will allow tailored exsolution of nanoparticles, which can be used for highly sophisticated catalyst design.

\section{Introduction}

The general formula of oxide-type perovskites is $\mathrm{ABO}_{3}$ (see Fig. 1a), where $A$ and $B$ are cations of different sizes. The smaller cations $B$ form corner-connected coordination octahedra with $\mathrm{O}$ atoms. Many different combinations of $A$ - and $B$-cations can be realized, and mixed cations on both $A$ and $B$ substructures are possible as well. Due to the wide range of properties resulting from this compositional flexibility, perovskites are a highly versatile class of materials - possible applications include sensors (Rahimi et al., 2019), electrode materials in fuel cells (Lu et al., 2016), photodetectors (Ahmadi et al., 2017), light-emitting diodes (Wang et al., 2018) and three-way converters (Keav et al., 2014). Moreover, these materials are promising candidates for a large variety of uses in catalysis (Hwang et al., 2017). It is possible to modify and fine-tune perovskites according to the desired properties, thus enabling rational material or catalyst design (Lindenthal et al., 2020). 
This outstanding feature has led to intense research on possible novel perovskites and their chemical composition. For example, Vieten et al. (2019) highlighted in a recent theoretical and experimental study how they can optimize the perovskite composition and structure for their application as oxygen carriers in thermochemical processes. A recent comprehensive overview on the design, synthesis, properties and applications of rare-earth-doped perovskites is given by Zeng et al. (2020). Also, for application as a cathode material in solid oxide fuel cells, the constant development of improved perovskites has been reported (Cascos et al., 2019). The application of rare-earth-based perovskites as suitable catalysts has already been investigated by many groups, for instance by $\mathrm{Lim}$ et al. (2018) for doped and undoped $\mathrm{LaFeO}_{3}$, and related materials. Furthermore, the group of Irvine reported a novel approach for exchanging $A$-site cations with $\mathrm{Ni}$ (Lee et al., 2020). They suggested that Ni exsolving from the $A$-site of a perovskite leads to the formation of catalytically highly active Ni nanoparticles, and thus to superior electrocatalysts for the oxygen evolution reaction.

A key requirement for excellent catalyst performance is the presence of catalytically active sites - typically, these consist of metal, alloy or oxide nanoparticles embedded in an oxide support material. Usually, catalytically active nanoparticles are prepared by deposition (Yates \& Campbell, 2011), impregnation (Gorte \& Vohs, 2009) or precipitation (Rousseau et al., 2010) techniques followed by catalyst activation prior to reactions via oxidation and reduction cycles. Often, these methods offer only limited control over the exact structure of the catalyst surface (Neagu et al., 2013). A recently emerging alternative method for preparing catalytically active sites uses nanoparticles grown in situ, directly from the oxide support itself (Neagu et al., 2013). Perovskites are able to incorporate catalytically highly active elements [e.g. Ni (Neagu et al., 2013; Kobsiriphat et al., 2010), Fe (Neagu et al., 2013; Opitz et al., 2015), Co (Adijanto et al., 2012), Cu (Neagu et al., 2013; Adijanto et al., 2012), Pt (Katz et al., 2012; Tanaka et al., 2006) and Pd (Tanaka et al., 2006; Katz et al., 2011; Eyssler et al., 2011)] - both as dopants or as main $B$ substructure components. Subsequently, these elements can be partially exsolved as nanoparticles under reducing conditions (Fig. 1b), leading to smaller and more finely distributed particles on the surface (Nishihata et al., 2002).

The mechanism and driving forces of nanoparticle exsolution from bulk perovskites have been the topics of multiple studies (Neagu et al., 2013; Oh et al., 2015; Thalinger et al., 2015; Haag et al., 2010). Factors that play a role in exsolution are the reducibility of the $B$-site cation or dopant element (Kwon et al., 2017), the oxygen partial pressure (connected also to the chemical potential of the $\mathrm{H}_{2} / \mathrm{H}_{2} \mathrm{O}$ gas phase) (Opitz et al., 2015) and the presence of oxygen vacancies (Neagu et al., 2013). Haag et al. (2010) proved by an in situ neutron diffraction study on $\mathrm{La}_{0.3} \mathrm{Sr}_{0.7} \mathrm{Fe}_{0.7} \mathrm{Cr}_{0.3} \mathrm{O}_{3-\delta}$ that by lowering the $p\left(\mathrm{O}_{2}\right)$ to $10^{-21.5} \mathrm{~atm}, \mathrm{Fe}$ nanoparticles were formed. A possible mechanism was proposed by Neagu et al. (2013), starting with the formation of oxygen vacancies upon reduction. The presence of these vacancies destabilizes the perovskite structure, locally causing the $B$-site species to exsolve in order to re-establish stoichiometry. For the morphological evolution of nanoparticles on the surface, Oh et al. (2015) provided an explanation by applying a simple energy-based model, taking into account the interplay between the surface free energy and the strain energy due to metal nucleates being included in the matrix. They showed an influence of these factors on the exsolution process using quantitative strain field modelling.

In this study, previous preliminary work on $\mathrm{Nd}_{0.6} \mathrm{Ca}_{0.4} \mathrm{FeO}_{3-\delta}$ and $\mathrm{Nd}_{0.6} \mathrm{Ca}_{0.4} \mathrm{Fe}_{0.9} \mathrm{Co}_{0.1} \mathrm{O}_{3-\delta}$ (Lindenthal et al., 2020) was extended to examine three additional materials, namely, $\mathrm{La}_{0.9} \mathrm{Ca}_{0.1} \mathrm{FeO}_{3-\delta}, \mathrm{La}_{0.6} \mathrm{Ca}_{0.4} \mathrm{FeO}_{3-\delta}$ and $\mathrm{Nd}_{0.9} \mathrm{Ca}_{0.1} \mathrm{FeO}_{3-\delta}$. For all five perovskite materials, the atomic and electronic structure, morphology and exsolution behaviour were investigated, with a focus on the effect of $A$-site doping with Ca. As reported previously by the authors, all five perovskites have been tested for their catalytic performance and are potential catalyst materials for high-temperature water-gas shift reactions (Popovic et al., 2020). The characterization was performed utilizing various experimental methods: in situ powder X-ray diffraction (XRD), scanning electron microscopy (SEM) combined with energy dispersive X-ray spectroscopy (EDX), and inductively coupled plasma-optical emission spectrometry (ICP-OES). The experimental results were supported by density functional theory (DFT) calculations. Additionally, exsolution properties were studied using in situ XRD and SEM/EDX.

\section{Experimental}

\subsection{Synthesis of doped perovskites}

The different perovskite powders were synthesized via the Pechini (1967) route. For this, the cations were mixed in the desired stoichiometric ratio using the following chemicals as

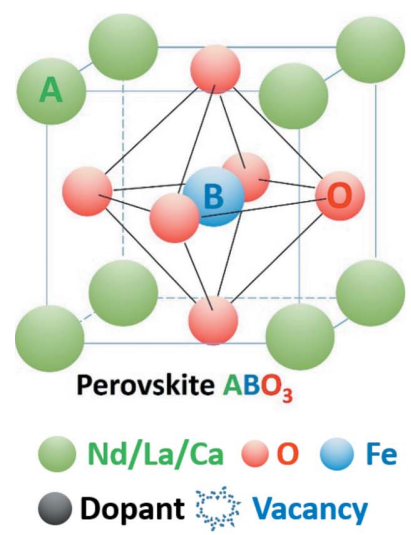

(a)

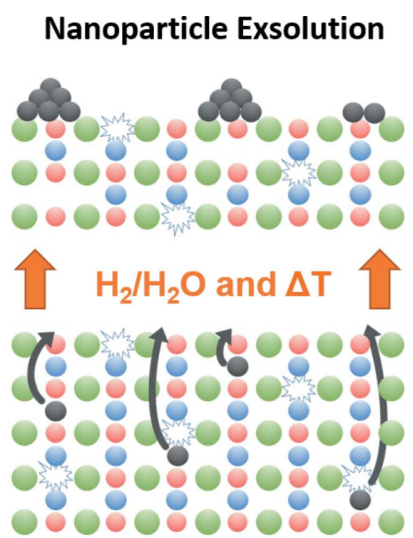

(b)
Figure 1

(a) The perovskite structure and (b) a schematic depiction of the exsolution process. In a wet hydrogen atmosphere and under heat treatment, additional oxygen vacancies are created and dopant atoms get reduced, resulting in their migration from the bulk to the surface, where they form nanoparticles. 
starting materials: $\mathrm{La}\left(\mathrm{CH}_{3} \mathrm{COO}\right)_{3} \cdot 1.5 \mathrm{H}_{2} \mathrm{O}(99.9 \%$, Alfa Aesar, Haverhill, MA, USA), $\mathrm{Nd}_{2} \mathrm{O}_{3}$ (99.9\%, Strategic Elements, Deggendorf, Germany), $\mathrm{CaCO}_{3}$ (99.95\%, Sigma-Aldrich, St Louis, MO, USA), Fe (99.5\%, Sigma-Aldrich, St Louis, MO, USA) and $\mathrm{Co}\left(\mathrm{NO}_{3}\right)_{3} \cdot 6 \mathrm{H}_{2} \mathrm{O}(99.999 \%$, Sigma-Aldrich, St Louis, MO, USA). The compounds were weighed accordingly and dissolved in $\mathrm{HNO}_{3}$ (doubly distilled, 65\%, Merck, Darmstadt, Germany). Cation complexes were formed by adding citric acid $(99.9998 \%$ trace metals pure, Fluka) in a molar ratio of 1.2 with respect to the cations. After evaporation of $\mathrm{H}_{2} \mathrm{O}$, the resulting gel was heated until self-ignition. The obtained powder was calcined for $3 \mathrm{~h}$ at $800^{\circ} \mathrm{C}$. After grinding, the resulting products were used for the (in situ) XRD, BET, ICP-OES and SEM studies.

For comparison, the commercial perovskite material $\mathrm{La}_{0.6} \mathrm{Sr}_{0.4} \mathrm{FeO}_{3-\delta}$ (LSF, lanthanum strontium ferrite, SigmaAldrich, St Louis, MO, USA) was measured using XRD.

\subsection{Characterization methods}

The powder XRD measurements were carried out on a PANalytical X'Pert Pro diffractometer (Malvern Panalytical, Malvern, UK) in Bragg-Brentano geometry using a mirror to single out the used $\mathrm{Cu} K \alpha_{1,2}$ radiation and an X'Celerator linear detector (Malvern Panalytical, Malvern, UK). For the in situ experiments, an Anton Paar XRK 900 chamber (Anton
Paar, Graz, Austria) was used. The pristine samples were pretreated in $\mathrm{O}_{2}$ at $600{ }^{\circ} \mathrm{C}$ for $30 \mathrm{~min}$. After cooling to room temperature, the atmosphere was changed to humidified $\mathrm{H}_{2}$ (using a bubbler at room temperature, $p=1$ bar, $\mathrm{H}_{2}: \mathrm{H}_{2} \mathrm{O} \simeq$ 32:1) and the temperature was increased gradually by $25^{\circ} \mathrm{C}$. At each step, an in situ XRD measurement was performed after waiting for $30 \mathrm{~min}$. The data were analysed using the HighScore Plus software (Degen et al., 2014) and the ICDD PDF-4+ 2019 database (ICDD, 2018).

To determine the actual composition of the synthesized samples, ICP-OES was used. The samples were digested in $\mathrm{HCl}$ and, after diluting, analysed with an iCAP 6500 ICP-OES spectrometer (Thermo Scientific, Waltham, MA, USA) equipped with a Meinhardt nebulizer and a cyclonic spray chamber (Glass Expansion, Port Melbourne, Australia). The observed signal intensities were converted into concentration units by means of external aqueous calibration. A detailed description of the measurement and quantification procedure is given in the supporting information ( $(\mathrm{S} 2)$.

The SEM experiments were carried out on a FEI QUANTA 250 FEG scanning electron microscope (FEI Company, Hillsboro, OR, USA) equipped with an EDAX Octane Elite $\mathrm{X}$-ray detector (EDAX Inc., Mahwah, NJ, USA) using (if not mentioned otherwise) an acceleration voltage of $5 \mathrm{kV}$ to achieve sufficient surface sensitivity. The images were recorded using secondary electrons.

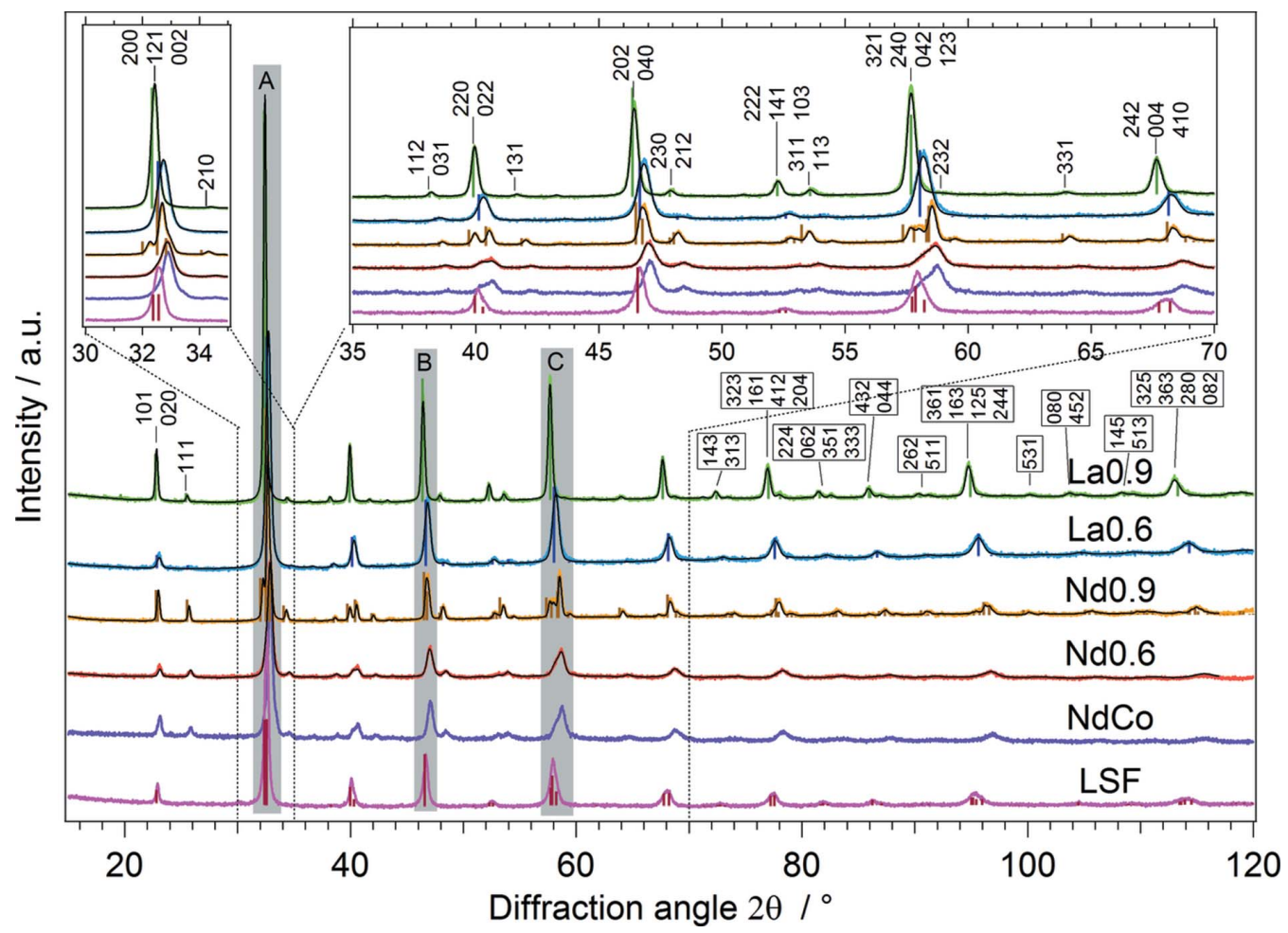

Figure 2

Diffractograms of the pristine samples. For comparison, simulated stick patterns of database structures of the same or similar materials are given $\left(\mathrm{La}_{0.9} \mathrm{Ca}_{0.1} \mathrm{FeO}_{3}\right.$ for $\mathrm{La} 0.9, \mathrm{La}_{0.67} \mathrm{Ca}_{0.33} \mathrm{FeO}_{3}$ for $\mathrm{La} 0.6, \mathrm{NdFeO}_{3}$ for $\mathrm{Nd} 0.9$ and LSF for LSF). The sticks coincide with the measured Bragg peaks, confirming a successful synthesis and phase purity. Additionally, the black lines are the simulated diffractograms after Rietveld refinement, agreeing very well with the measured intensities. The $h \mathrm{kl}$ indices of the reflections that contribute most to the respective (group of) Bragg peaks are given. Please note that this list is not complete (especially for high angles). The largest Bragg peaks (A-C) are highlighted in grey (see Table 2 for their positions). 


\subsection{DFT calculations}

Spin-polarized DFT (DFT+U) calculations were carried out with the software package WIEN2k (Blaha et al., 2018), which uses the augmented plane wave plus the local orbital method (Karsai et al., 2017), treating all electrons in a full potential [FP-(L)APW+lo]. To treat exchange-correlation, the GGA functional PBE (Perdew et al., 1996) was chosen and a Hubbard-U (Anisimov et al., 1991) was added to properly consider localized electrons: $\mathrm{U}$ was always included for $\mathrm{Fe} 3 d$ electrons and for $\mathrm{Nd} 4 f$ electrons for the $\mathrm{Nd}$ materials. For all materials, $U_{\text {eff }}$ was set at $4 \mathrm{eV}$ (Kraushofer et al., 2018; Nilsson et al., 2013), both for $\mathrm{Fe}$ and $\mathrm{Nd}$ states.

The simulation of $\mathrm{Nd}_{0.5} \mathrm{Ca}_{0.5} \mathrm{FeO}_{3}$ has been described in a previous study (Lindenthal et al., 2020). Here, the following computational parameters have been used: structure models for the calculations were created using the experimentally found lattice parameters (XRD data are presented in $\$ 3.2$; see Table 3 and Fig. 2). The unit cells of the bulk materials contain four formula units of $\mathrm{LaFeO}_{3}$ and $\mathrm{NdFeO}_{3}$, respectively. These unit cells ( $c f$. Fig. 3) were used directly to simulate $\mathrm{La}_{0.5} \mathrm{Ca}_{0.5} \mathrm{FeO}_{3}$ and $\mathrm{Nd}_{0.5} \mathrm{Ca}_{0.5} \mathrm{FeO}_{3}$ (corresponding to a $50 \%$ doping), where two of the four $\mathrm{La} / \mathrm{Nd}$ atoms were substituted by Ca. Additionally, $(\sqrt{2} \times 1 \times \sqrt{2}) R 45$ supercells (see Figs. S4 and S5 in the supporting information) were set up, resulting in cells with eight formula units, thus enabling simulations of $\mathrm{La}_{0.875} \mathrm{Ca}_{0.125} \mathrm{FeO}_{3}$ and $\mathrm{Nd}_{0.875} \mathrm{Ca}_{0.125} \mathrm{FeO}_{3}$ (corresponding to a $12.5 \%$ doping) by substituting one $\mathrm{La} / \mathrm{Nd}$ atom with $\mathrm{Ca}$. The $\mathrm{O}$ substructure was chosen fully occupied to emulate materials under highly oxidizing conditions to reduce computational cost. From a defect chemical point of view, this corresponds to charge compensation by electron holes rather than oxygen vacancies. This assumption is further justified by the fact that the materials were prepared in air and cooled slowly, which means that the expected vacancy concentration is low. Consequently, the effects of Ca doping are assumed to play a more important role than oxygen vacancies. The atomic positions were optimized until residual forces were below $1 \mathrm{mRy} /$ Bohr for all cases.

While La could be treated nonmagnetically, both $\mathrm{Nd}$ and $\mathrm{Fe}$ exhibit magnetic ordering: Fe atoms are arranged in a type $\mathrm{G}$ (Wollan \& Koehler, 1955) antiferromagnetic substructure (Sławiński et al., 2005), where the spin (up and down, respectively) of any given $\mathrm{Fe}$ atom orients antiparallel to all next nearest neighbours. Experimental studies have shown long-range antiferromagnetic ordering of the $\mathrm{Nd}$ atoms in $\mathrm{NdFeO}_{3}$ at very low temperatures of about $-271{ }^{\circ} \mathrm{C}$ (Sławiński et al., 2005; Bartolomé et al., 1997). However, to reduce computational cost, a ferromagnetic set-up was used in initial simulations, since ferromagnetic $\mathrm{Nd}$ ordering only weakly affects the moments of other atoms (in the order of $0.01 \mu_{\mathrm{B}}$ ). The results of ferromagnetic calculations (optimized positions) have been used to check structures with antiferromagnetically ordered $\mathrm{Nd}$ atoms - no significant changes of the residual forces could be observed (all forces remained below $1.5 \mathrm{mRy} / \mathrm{Bohr}$ ). Therefore, no additional calculations with antiferromagnetic $\mathrm{Nd}$ have been performed.
Atomic sphere radii for all calculations were chosen as follows: 2.20, 1.92 and $1.60 \mathrm{Bohr}$ for $\mathrm{La} / \mathrm{Nd} / \mathrm{Ca}, \mathrm{Fe}$ and $\mathrm{O}$, respectively. The basis set size is given by the plane wave cutoff $R K_{\max }$, where the smallest atomic sphere radius is $R$ and $K_{\max }$ is the largest vector in reciprocal space to be considered. $R K_{\max }=9$ (La cases) and $R K_{\max }=8$ (Nd cases) were used. Brillouin-zone integrations of the doped materials were done on an $8 \times 6 \times 8$ Monkhorst-Pack k-mesh (Monkhorst \& Pack, 1976 ) for $50 \%$ doping and a $6 \times 6 \times 6$ mesh for $12.5 \%$ doping.

The results of the $A$-site-doped materials were compared with those of undoped bulk $\mathrm{LaFeO}_{3}$ (Falcón et al., 1997) and $\mathrm{NdFeO}_{3}$ (Streltsov \& Ishizawa, 1999), which were obtained using the same parameters as the respective $50 \%$-doped perovskites.

\section{Results}

\subsection{Choice of materials}

The studied perovskites should fulfil several prerequisites for their intended application in catalysis. First, it should be possible to prepare them reproducibly, and they should be
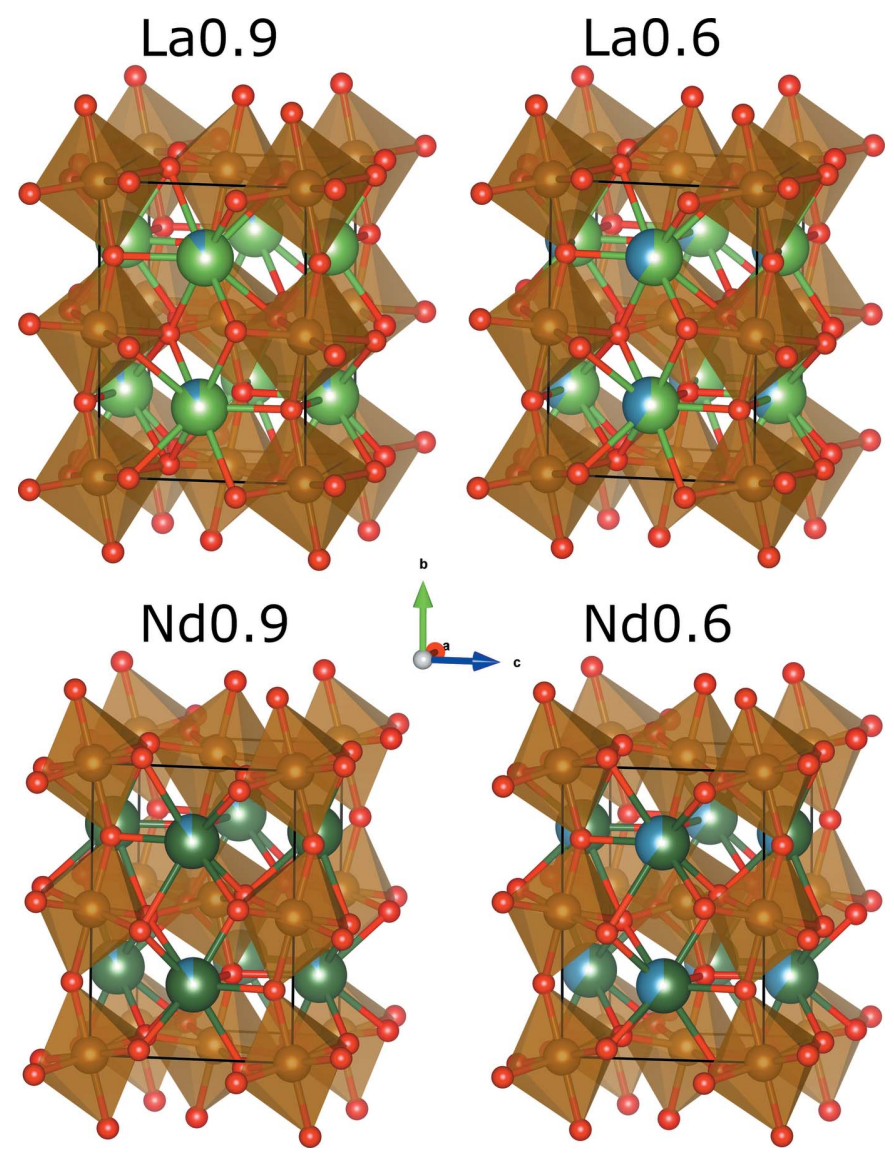

Figure 3

Perovskite structures of the $A$-site-doped materials without $B$-site doping, found by Rietveld refinement. All synthesized perovskites are isotypic and crystallized in the orthorhombic space group Pnma (No. 62). The structures are related to the ideal cubic perovskite structure, but with tilted Fe coordination octahedra. Differences of the structures between the various materials were found only in the extent of the tilting and the distortion of the octahedra. 
Table 1

Investigated materials with different $A$ - and $B$-site compositions.

The following notations will be used. The $A$-site consists of La or Nd, doped with either $\mathrm{Ca}$ (synthesized materials) or $\mathrm{Sr}$ (commercially available material). For $\mathrm{Ca}$, the amount of doping is varied. The $B$-site consists of $\mathrm{Fe}$, with possible doping with Co.

\begin{tabular}{|c|c|c|c|c|c|}
\hline \multirow{2}{*}{$\frac{\text { Nominal }}{\mathrm{La}_{0.9} \mathrm{Ca}_{0.1} \mathrm{FeO}_{3-\delta}}$} & \multicolumn{2}{|c|}{$A$-site } & \multicolumn{2}{|c|}{$B$-site } & \multirow{2}{*}{$\frac{\text { Notation }}{\text { La0.9 }}$} \\
\hline & $\mathrm{La}: \mathrm{Ca}$ & $9: 1$ & $\mathrm{Fe}$ & & \\
\hline $\mathrm{La}_{0.6} \mathrm{Ca}_{0.4} \mathrm{FeO}_{3-\delta}$ & $\mathrm{La}: \mathrm{Ca}$ & $6: 4$ & $\mathrm{Fe}$ & & $\mathrm{La} 0.6$ \\
\hline $\mathrm{Nd}_{0.9} \mathrm{Ca}_{0.1} \mathrm{FeO}_{3-\delta}$ & $\mathrm{Nd}: \mathrm{Ca}$ & $9: 1$ & $\mathrm{Fe}$ & & $\mathrm{Nd} 0.9$ \\
\hline $\mathrm{Nd}_{0.6} \mathrm{Ca}_{0.4} \mathrm{FeO}_{3-\delta}$ & $\mathrm{Nd}: \mathrm{Ca}$ & $6: 4$ & $\mathrm{Fe}$ & & Nd0.6 \\
\hline $\mathrm{Nd}_{0.6} \mathrm{Ca}_{0.4} \mathrm{Fe}_{0.9} \mathrm{Co}_{0.1} \mathrm{O}_{3-\delta}$ & $\mathrm{Nd}: \mathrm{Ca}$ & $6: 4$ & Fe:Co & $9: 1$ & $\mathrm{NdCo}$ \\
\hline $\mathrm{La}_{0.6} \mathrm{Sr}_{0.4} \mathrm{FeO}_{3-\delta}$ & $\mathrm{La}: \mathrm{Sr}$ & $6: 4$ & $\mathrm{Fe}$ & & LSF \\
\hline
\end{tabular}

stable. They should show a good performance as catalysts and they should be able to incorporate catalytically active elements, which can be exsolved upon reduction (Neagu et al., 2013) to form catalytically highly active nanoparticles on the surface. In particular, the exsolution of the most catalytically active elements under reasonable conditions is preferred. Lastly, to be able to use them as electrode materials in electrochemical cell design (e.g. in fuel cells or to apply a polarization for in situ fine-tuning of the exsolution process), they should have mixed ionic electronic conductivity (MIEC) (Opitz et al., 2018).

To achieve these properties, the elements for the $A$ - and $B$-sites of the $A B \mathrm{O}_{3}$ perovskites have to be chosen appropriately. Furthermore, both sites can be doped or a nonstoichiometry can be introduced, with a deficiency on either of the sites. The starting point for the choice of composition for our materials was the commercially available material $\mathrm{La}_{0.6} \mathrm{Sr}_{0.4} \mathrm{FeO}_{3-\delta}$ (LSF). This type of perovskite is widely used by different research groups and therefore extensive reference data for defect chemistry (Kuhn et al., 2011; Schmid et al., $2018 b$ ) and electrochemical properties (Preis et al., 2004; Schmid et al., 2018a; Søgaard et al., 2007) exists. The replacement of $\mathrm{La}^{3+}$ with the acceptor dopant $\mathrm{Sr}^{2+}$ leads to a charge imbalance in the perovskite structure, giving rise to a complex defect chemistry. Depending on oxygen partial pressure and temperature, this charge mismatch is compensated by either oxygen vacancies (denoted by $\mathrm{O}_{3-\delta}$ in the formula) or mixed $\mathrm{Fe}^{4+}, \mathrm{Fe}^{3+}$ and $\mathrm{Fe}^{2+}$ valence states. These defects are responsible for the required MIEC properties (Yoon et al., 2009).

Additionally, the $A$-site elements and doping amount influence the thermochemical stability and exsolution behaviour (Neagu et al., 2015). A-site cations with smaller ionic radii (e.g. Ca and $\mathrm{Nd}$ ) lead to distortions in the perovskite structure, affecting its stability (see $\$ 3.2$ ). The stability is also affected by the chemical properties of the elements, e.g. reducibility and segregation tendency. Thus, varying the $A$-site composition allows tuning of the exsolution properties. Therefore, we exchanged the Sr doping for Ca with the aim of increasing the stability and investigated the effect of $\mathrm{Ca}$ doping (40 and $10 \%$ of $A$-site ions). Also, we exchanged $\mathrm{La}$ with $\mathrm{Nd}$ to increase the structural distortions and facilitate XPS studies. Since a future goal is to exsolve metallic Ni particles from these host perovskites (respective work is in
Table 2

Compositions and PDF numbers of the database structures used as a reference pattern in Fig. 2 and as a starting point for Rietveld refinement.

The positions of the maxima of the largest Bragg peaks are given (the notation refers to the markings in Fig. 2).

\begin{tabular}{lllllll}
\hline & \multicolumn{2}{c}{ Reference structure } & & \multicolumn{3}{c}{ Main Bragg peak positions $2 \theta\left(^{\circ}\right)$} \\
\cline { 2 - 3 } Sample & Composition & PDF No. & & $A$ & $B$ & $C$ \\
\hline $\mathrm{La} 0.9$ & $\mathrm{La}_{0.9} \mathrm{Ca}_{0.1} \mathrm{FeO}_{3}$ & $01-082-9272$ & & 32.4 & 46.4 & 57.7 \\
$\mathrm{La} 0.6$ & $\mathrm{La}_{0.67} \mathrm{Ca}_{0.33} \mathrm{FeO}_{3}$ & $04-017-9772$ & & 32.7 & 46.8 & 58.2 \\
$\mathrm{Nd} 0.9$ & $\mathrm{NdFeO}_{3}$ & $04-014-5430$ & 32.7 & 46.9 & 58.5 \\
$\mathrm{Nd} 0.6$ & $\mathrm{NdFeO}_{3}$ & $04-014-5430$ & 32.9 & 47.1 & 58.7 \\
$\mathrm{NdCo}$ & & & 32.9 & 47.1 & 58.8 \\
LSF & $\mathrm{La}_{0.6} \mathrm{Sr}_{0.4} \mathrm{FeO}_{3}$ & $04-007-6517$ & & 32.6 & 46.7 & 57.9 \\
\hline
\end{tabular}

progress), the overlap of Ni $2 p$ and La $3 d$ lines can be avoided by exchanging $\mathrm{La}$ with $\mathrm{Nd}$, thus allowing an easier XPS characterization of such an Ni-containing exsolution catalyst. Furthermore, rare earth materials are generally known for their catalytic activity, and both elements were reported in the literature to have a promotional effect on catalytic reactions (e.g. for water-gas shift) (LeValley et al., 2014).

With these prerequirements, the following materials, $\mathrm{La}_{0.9} \mathrm{Ca}_{0.1} \mathrm{FeO}_{3-\delta}(\mathrm{La} 0.9), \mathrm{La}_{0.6} \mathrm{Ca}_{0.4} \mathrm{FeO}_{3-\delta}(\mathrm{La} 0.6), \mathrm{Nd}_{0.9} \mathrm{Ca}_{0.1^{-}}$ $\mathrm{FeO}_{3-\delta}(\mathrm{Nd} 0.9)$ and $\mathrm{Nd}_{0.6} \mathrm{Ca}_{0.4} \mathrm{FeO}_{3-\delta}(\mathrm{Nd} 0.6)$, were synthesized. In addition, $B$-site doping with the catalytically promising element $\mathrm{Co}$ was tested. For this purpose, the material $\mathrm{Nd}_{0.6} \mathrm{Ca}_{0.4} \mathrm{Fe}_{0.9} \mathrm{Co}_{0.1} \mathrm{O}_{3-\delta}(\mathrm{NdCo})$ was investigated. The specific $A$-site composition for the doped material was chosen, because it proved to be the most stable with respect to exsolution. A stable host perovskite should favour the preferential exsolution of the dopant metal, making it possible to produce only pure Co particles instead of mixtures with $\mathrm{Fe}$ particles or alloys with Fe. Table 1 gives an overview of all the materials that were synthesized. Also, doping with $\mathrm{Ni}$ was considered, but as it could not be synthesized phase pure, this attempt was dropped (see $\S \mathrm{S} 1$ in the supporting information for details).

The real composition of the samples after synthesis was determined by ICP-OES; Table S2 in the supporting information summarizes the results. The composition derived by ICP-OES deviates from the desired nominal stoichiometry by a maximum of $10 \%$, which confirms that the respective synthesis was successful. In all materials, there is a slight $A$-site deficiency. This deficiency is almost negligible for the samples with $\mathrm{La}$, but slightly more prominent for those with $\mathrm{Nd}$. However, an $A$-site deficiency should enhance the exsolution properties, because exsolution re-establishes the stoichiometry within the material in such a case (Neagu et al., 2013). Therefore, the deviations from the nominal compositions should be beneficial for the later catalytic application of the investigated materials.

\subsection{Structure characterization}

To determine the crystal structure of the freshly synthesized samples, powder X-ray diffractograms of the pristine samples were recorded (see $\S \mathrm{S} 3$ of the supporting information for experimental tables). For comparison, the commercial mate- 
rial LSF is also displayed. The results for the samples are displayed in Fig. 2. The diffractograms of $\mathrm{La} 0.9$ and $\mathrm{La} 0.6$ showed similarities in appearance, differing mostly in the exact position of the Bragg peaks and their intensity. Nd0.9 and $\mathrm{Nd} 0.6$ showed a very similar diffraction pattern to the samples with $\mathrm{La}$, but some reflections were split and, again, the positions and intensities varied. These similarities of the diffractograms suggested that all the materials had similar structures (as is expected, because they were all perovskite materials from the same family). Also, LSF shows a related pattern sharing the main peaks with the synthesized materials, but the absence of peak splitting (i.e. of weaker reflections) indicates a changed symmetry of the LSF unit cell.

In Fig. 2, the measured diffractograms are overlaid with simulated stick patterns of the perovskite structures taken from a database of the same materials (if available, as for La0.9 and LSF) or similar materials (with a slightly different $A$-site composition). The database structures used were taken from the ICDD PDF-4+ 2019 database (ICDD, 2018) and are listed in Table 2. For Nd0.6, no database entry for a material with a similar $A$-site composition existed; thus, no stick pattern is shown. The measured Bragg peaks coincided well with the sticks, especially for La0.9 and LSF. For the other samples, when only a similar material was used as a reference, a slight shift could be observed. Also, no additional peaks which are not part of the stick patterns were visible in the diffractograms. This confirmed that the syntheses were successful and perovskite structures were obtained. Furthermore, the absence of additional peaks proves the phase purity of the synthesized materials.

When comparing the materials with Ca doping to Sr-doped LSF (Fossdal et al., 2004), the latter shows fewer Bragg peaks in the diffractograms (which is also reflected in the stick patterns). This is due to the different space group of these materials. The Ca-doped perovskites crystallized in the orthorhombic space group Pnma (No. 62), while LSF has the rhombohedral space group $R \overline{3} c$ (No. 167). The differing symmetry of a rhombohedral lattice results in fewer peaks in the diffractogram. The appearance or absence of those peaks could be used to differentiate between the two lattice symmetries.

For an exact determination of the crystal structures, Rietveld refinements were performed with the HighScore Plus software (Degen et al., 2014), using the whole $2 \theta$ range 15 $120^{\circ}$. For this, the same structures that were used for the reference stick patterns served as a starting point for the respective phase used in the refinement (see Table 2). For the materials with a differing $A$-site composition, the composition of the starting material was corrected before the refinement. Next, the scale factor, the unit-cell parameters and the profile variables (Caglioti parameters) of the phase were refined. Finally, the atomic positions that are not symmetry-fixed were released in a last refinement step. Geometric differences between the starting structures and the final refined structures are listed in Tables S7 and S8 of the supporting information. The simulated intensity profiles after the Rietveld refinement are shown in Fig. 2 as thin black lines. They agree very well
Table 3

Results of the Rietveld refinement.

The quality parameter $\chi^{2}$ quantifies the agreement of the calculated to the measured diffractogram (values closer to 1 suggest good refinement results). Additionally, the unit-cell parameters $a, b$ and $c$, and the unit-cell volume $V$ of the orthorhombic unit cells of the perovskite structures determined by Rietveld refinement are given. LSF data [taken from PDF \#04-007-6517) are shown for comparison (rounded to the same number of digits)] - the volume is normalized to four formula units (as in the orthorhombic cell).

\begin{tabular}{|c|c|c|c|c|c|}
\hline \multirow[b]{2}{*}{ Sample } & \multirow[b]{2}{*}{$\chi^{2}$} & \multicolumn{3}{|c|}{ Cell parameters } & \multirow{2}{*}{$\begin{array}{l}\text { Cell volume } \\
V\left(\AA^{3}\right)\end{array}$} \\
\hline & & $a(\AA)$ & $b(\AA)$ & $c(\AA)$ & \\
\hline $\mathrm{La} 0.9$ & 2.575 & $5.558(1)$ & 7.837 (2) & $5.547(1)$ & 241.7 (1) \\
\hline $\mathrm{La} 0.6$ & 2.197 & $5.506(4)$ & $7.776(6)$ & $5.530(4)$ & $236.8(3)$ \\
\hline Nd0.9 & 1.894 & $5.568(2)$ & $7.757(3)$ & $5.451(2)$ & $235.4(2)$ \\
\hline $\mathrm{Nd} 0.6$ & 1.375 & $5.533(8)$ & $7.73(1)$ & $5.449(8)$ & $233(1)$ \\
\hline LSF & - & 5.527 & 5.527 & 13.421 & 236.7 \\
\hline
\end{tabular}

Table 4

Structural comparison of the synthesized perovskites without $B$-site doping and of commercially available LSF.

The structures were characterized by the space group, the Glazer angles $a$ and $b$, as well as by the angles $\gamma$ (with respect to the [010] direction) and $\delta$ (with respect to the [101] direction), which have been introduced for comparison with the DFT results (see also Fig. S3 in the supporting information).

\begin{tabular}{llrlll}
\hline Sample & Space group & $a\left(^{\circ}\right)$ & $b\left(^{\circ}\right)$ & $\gamma\left(^{\circ}\right)$ & $\delta\left(^{\circ}\right)$ \\
\hline La0.9 & Pnma $(62)$ & 7.47 & 8.69 & 11.72 & 10.94 \\
La0.6 & Pnma (62) & 8.16 & 8.66 & 12.01 & 11.85 \\
Nd0.9 & Pnma (62) & 10.18 & 8.73 & 14.27 & 13.40 \\
Nd0.6 & Pnma (62) & 10.84 & 6.22 & 14.26 & 12.96 \\
LSF & R $\overline{3} c(167)$ & 4.84 & - & - & - \\
\hline
\end{tabular}

with the measured intensities. This agreement was also quantified with the quality parameter $\chi^{2}$ [this is related to the goodness-of-fit as given by Toby (2006); see Table 3 for the determined values - values of $\chi^{2}$ closer to 1 suggest good refinement results (further agreement indices are listed in the experimental tables - see supporting information). For all samples, this parameter is below 3 , indicating a reasonable fit. For the samples with Nd, the fit is even better than for those with $\mathrm{La}$. In all cases, this supports that the determined structure models were correct.

There was no refinement performed for the Co-doped material. Its diffractogram is almost identical to that of Nd0.6; all the Bragg peaks visible for $\mathrm{Nd} 0.6$ appear also for $\mathrm{NdCo}$, with nearly the same positions, intensities and shapes. Therefore, we concluded that the small amount of doping did not have a strong influence on the structure and assumed that the structure found for Nd0.6 sufficiently well describes also that for NdCo. Any differences between the structures obtained with Rietveld refinement would be within the error of the method.

The structures found with Rietveld refinement are displayed in Fig. 3 and the corresponding unit-cell parameters and unit-cell volumes are given in Table 3. All synthesized doped perovskites are isotypic. Their structures can be derived from the ideal cubic perovskite structure (see Fig. 1a) through a tilting of the Fe coordination octahedra, resulting in reduced symmetry and an orthorhombic structure. As in the ideal 
structure, the oxygen anions are at the corners of these octahedra. The octahedra are connected via these corners, forming a network with the $A$ cations in between. Similarly, LSF has a rhombohedral lattice instead of the cubic one, induced by another type of octahedral tilting. These structures and the differences between the various materials are discussed further below.

The reason for the deviation from the cubic structure can be found in the size of the elements involved. Goldschmidt (1926) gives a criterion for the ideal ratios of the ionic radii. To compensate for too-small $A$-site cation radii (Goldschmidt tolerance factor below 1), in the investigated materials distortions and tilting of the $\mathrm{Fe}-\mathrm{O}$ octahedra occur. $\mathrm{As} \mathrm{Sr}^{2+}$ is larger than $\mathrm{Ca}^{2+}$, a different tilt system appears in LSF. The two possible symmetries are compared in detail in Fig. S3 (see supporting information). For both symmetries, the alternate tilting of the octahedra can be seen very well, but the tilting patterns are different for the different symmetries. According to Glazer's classification (Glazer, 1972), the tilt system of the orthorhombic structure is given as $a^{-} b^{+} a^{-}$and as $a^{-} a^{-} a^{-}$for LSF (where $a$ and $b$ are tilting angles). To allow comparison with the DFT results, additional angles $\gamma$ and $\delta$ between $\mathrm{Fe}-\mathrm{O}$
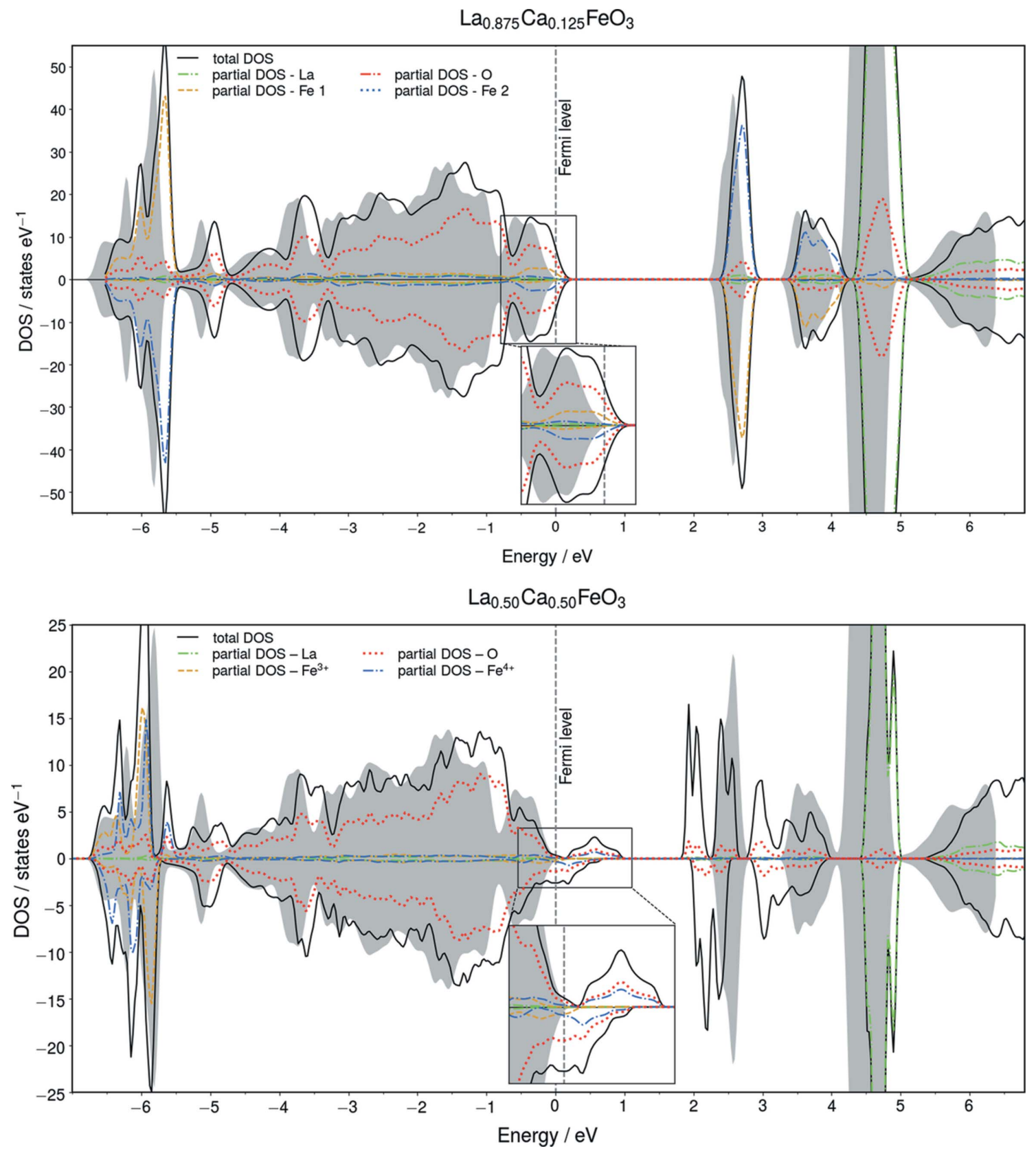

Figure 4

Total (black solid lines) and partial (dashed coloured lines) DOS for $\mathrm{La}_{0.875} \mathrm{Ca}_{0.125} \mathrm{FeO}_{3}$ (top) and for $\mathrm{La}_{0.5} \mathrm{Ca}_{0.5} \mathrm{FeO}_{3}$ (bottom). The dashed vertical lines represent the Fermi levels (set to $0 \mathrm{eV}$ ). The total DOS for the undoped bulk is shown as a grey area for reference. The insets show the region around the Fermi level. States above the $x$ axis (positive values) correspond to states of spin-up electrons, while states below the $x$ axis (negative values) correspond to states of spin-down electrons. For $\mathrm{Fe}^{3+}$ and $\mathrm{Fe}^{4+}$ in the lower figure, only one spin is shown and the antiferromagnetic counterparts have been omitted. 
and the [010] and [101] directions, respectively, are determined also (the angles are given in Table 4 and marked in Fig. S3 of the supporting information). This is necessary due to magnetism and doping, consequently leading to calculations done in low symmetry space groups $(P 1$ and $P \overline{1})$ for which no Glazer tilt systems are defined.

In an ideal cubic structure, all considered angles $a, b, \gamma$ and $\delta$ should be $0^{\circ}$. Tilting of the octahedra increases these angles. Thus, larger angles mean a more pronounced distortion. The perovskites containing $\mathrm{La}^{3+}$, which is larger than $\mathrm{Nd}^{3+}$, have a lower Glazer angle $a$ and less tilting than the materials with $\mathrm{Nd}^{3+}$. The same trend was confirmed when looking at LSF with the larger $\mathrm{Sr}^{2+}$ ions instead of $\mathrm{Ca}^{2+}$ ions. Consequently, LSF has the lowest observed distortion. In general, the tilting is stronger in the orthorhombic structures, induced by the smaller $A$-site cations and the higher non-ideality. The Glazer angle $b$ does not change in the case of $\mathrm{Nd} 0.9$, but decreases significantly for Nd0.6 (compared to the respective La materials). However, the overall distortion of Nd0.6 is still larger than in La0.6 (since the difference in angle $a$ is larger than in angle $b$ ). Additionally, it was observed that in the case of $\mathrm{La}$

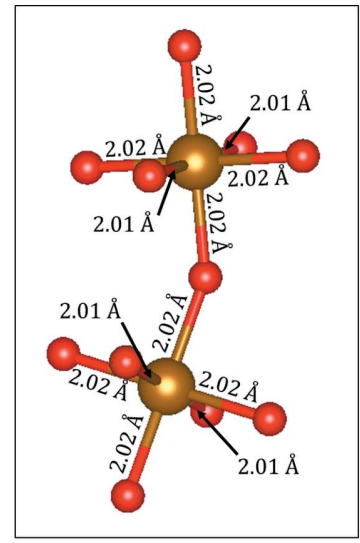

(a)

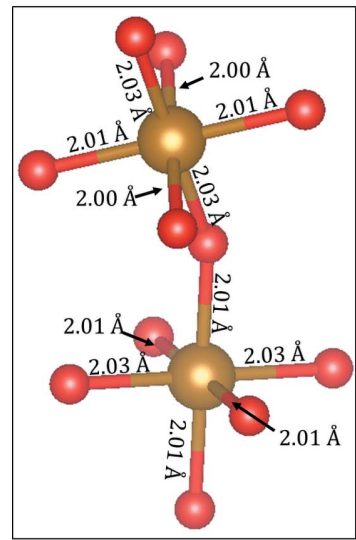

(c)

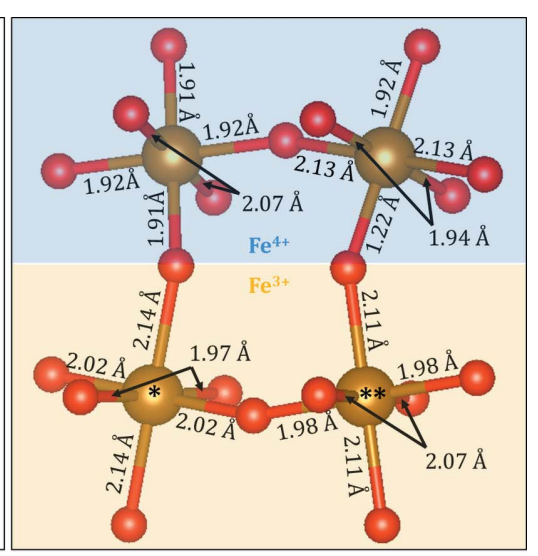

(b)

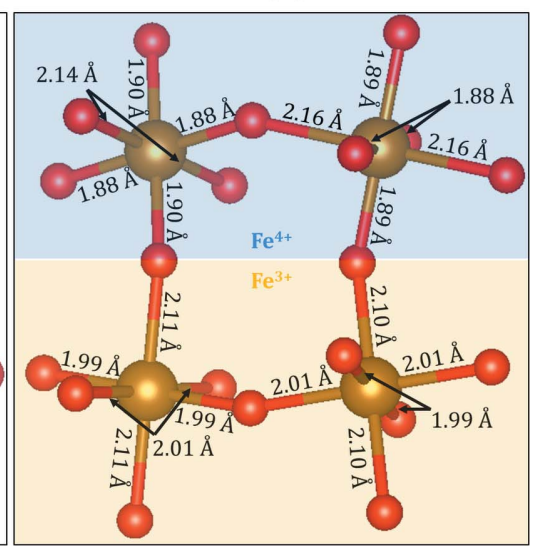

(d)
Figure 5

Calculated distances in the $\mathrm{Fe}-\mathrm{O}$ coordination octahedra for $(a) \mathrm{LaFeO}_{3}$, (b) $\mathrm{La}_{0.5} \mathrm{Ca}_{0.5} \mathrm{FeO}_{3}$, (c) $\mathrm{NdFeO}_{3}$ and $(d) \mathrm{Nd}_{0.5} \mathrm{Ca}_{0.5} \mathrm{FeO}_{3}$. In (b) two $\mathrm{Fe}$ atoms are marked with asterisks, since they exhibit different $\mathrm{Fe}-\mathrm{O}$ distortions: the left $\mathrm{Fe}$ atom (*) behaves similarly to both $\mathrm{Fe}$ atoms of $\mathrm{Nd}_{0.5} \mathrm{Ca}_{0.5} \mathrm{FeO}_{3}$, showing elongation in only one direction, while the right $\mathrm{Fe}$ atom (**) differs from the $\mathrm{Nd}$-analogue with $\mathrm{Fe}-\mathrm{O}$ distances elongated in two directions. perovskite, a higher $\mathrm{Ca}$ content increases the angle $a$, while angle $b$ remains unchanged. For the Nd perovskites, angle $a$ increases also with increasing Ca content; however, angle $b$ decreases.

The DFT angles $\gamma$ and $\delta$ considered for comparison are averaged due to local distortions present during the calculations (the individual angles for one averaged value vary by up to $4^{\circ}$ ). In general, the calculated averages are larger than the respective experimental values (with the exception of $\mathrm{Nd} 0.6$ ) and agree within $10 \%$ (with the exception of La0.9, where the deviation is larger by up to $20 \%$ ). However, the trend of increasing distortion when exchanging $\mathrm{La}$ with $\mathrm{Nd}$ was observed as well.

The volume $V$ reflects the sizes of the ions in the respective perovskite, as well as the extent of the tilting (reduced volume corresponds to a higher Glazer angle $a$, as tilting reduces the respective unit-cell parameters). When exchanging $\mathrm{La}^{3+}$ with the smaller $\mathrm{Nd}^{3+}, V$ is reduced. Larger amounts of Ca doping increase the amount of $\mathrm{Fe}^{4+}$, which replaces the larger $\mathrm{Fe}^{3+}$ (which is consistent with DFT results). For La0.9 and La0.6, this effect is combined with the replacement of $\mathrm{La}^{3+}$ with the slightly smaller $\mathrm{Ca}^{2+}$, thus also resulting in a lower $V$. For $\mathrm{Nd} 0.9$ and $\mathrm{Nd} 0.6$, replacing $\mathrm{Nd}^{3+}$ with $\mathrm{Ca}^{2+}$ leads to larger $A$-site cations on average, but the volume still decreases with the higher amount of Ca doping. In this case, the effect of changed $B$-site cation size due to a changed oxidation state seems predominant. However, the difference in $V$ between $\mathrm{Nd} 0.9$ and $\mathrm{Nd} 0.6$ is smaller than between $\mathrm{La} 0.9$ and $\mathrm{La} 0.6$. The volume of LSF is not comparable due to the different synthesis - it has a slightly lower volume per formula unit than La0.6, even though $\mathrm{Sr}^{2+}$ is larger than $\mathrm{Ca}^{2+}$ and the Glazer angles are smaller.

\subsection{DFT calculations}

DFT calculations were performed to further investigate the effect that $A$-site doping with $\mathrm{Ca}$ has on the atomic and electronic structures of the perovskites. In doped $\mathrm{NdFeO}_{3}$, this effect has been studied previously by Wang et al. (2015), treating the Nd- $4 f$ electrons in the frozen core approximation. In a previous study by the authors (Lindenthal et al., 2020), full potential [FP-(L)APW+lo] calculations for $\mathrm{Nd}_{0.5} \mathrm{Ca}_{0.5} \mathrm{FeO}_{3}$ were carried out. Here, we extend these calculations to $\mathrm{Nd}_{0.875} \mathrm{Ca}_{0.125} \mathrm{FeO}_{3}, \mathrm{La}_{0.875} \mathrm{Ca}_{0.125} \mathrm{FeO}_{3}$ and $\mathrm{La}_{0.5} \mathrm{Ca}_{0.5} \mathrm{FeO}_{3}$ for comparison.

The densities of states (DOS) of all simulated perovskites were calculated. Both undoped bulk materials $\mathrm{LaFeO}_{3}$ and $\mathrm{NdFeO}_{3}$ are insulating (each with a calculated band gap of $2.4 \mathrm{eV}$ ), while all doped materials show metallic behaviour. With Ca doping, the DOS gets shifted to the right with respect to the Fermi level due to the reduced number of valence electrons (exchanging $\mathrm{La}^{3+} / \mathrm{Nd}^{3+}$ with $\mathrm{Ca}^{2+}$ reduces this number, leading to an electron hole), i.e. states that are occupied in the bulk are empty in the doped material. The nature of the now-empty states together with the magnetic moments of the Fe atoms can be used to assess the effect of $\mathrm{Ca}$ doping on the electronic structure. 
In the case of $\mathrm{La}_{0.875} \mathrm{Ca}_{0.125} \mathrm{FeO}_{3}$ (Fig. 4, top), the DOS remains qualitatively the same compared to the bulk $\mathrm{LaFeO}_{3}$, apart from being shifted to the right. Both O- $p$ and Fe- $d$ states contribute to the empty states above the Fermi level, suggesting a delocalization of the electron hole over many $\mathrm{O}$ atoms (since there are $24 \mathrm{O}$ atoms in the unit cell, which contribute to those states), as well as Fe atoms. The magnetic moments of Fe do not change (the changes are below $0.05 \mu_{\mathrm{B}}$ per atom), which indicates no change of oxidation state.

$\mathrm{La}_{0.5} \mathrm{Ca}_{0.5} \mathrm{FeO}_{3}$ (Fig. 4, bottom) shows further differences. While there is still a large contribution of the O-p states to the states above the Fermi level (indicating delocalization of the electron hole), two different 'types' of Fe atoms can be seen now: (i) $\mathrm{Fe}^{3+}$ with hardly any contribution to the empty states above the Fermi level and (ii) $\mathrm{Fe}^{4+}$ with empty states (both spin-up and spin-down). Those empty states of $\mathrm{Fe}^{4+}$ are evidence for partial oxidation, which is also supported by the reduction of the magnetic moments of these atoms (the spinup and spin-down moments change from 4.0 to 3.4 and $3.6 \mu_{\mathrm{B}}$, respectively; this change of the moment occurs due to changes in the occupation of the respective Fe states), which turn out to be $\mathrm{Fe}$ with slightly larger $\mathrm{Fe}-\mathrm{Ca}$ distances. The moments of spin-up $\mathrm{Fe}^{3+}$ do not change with doping and spin-down $\mathrm{Fe}^{3+}$ exhibits a very slight reduction of about $0.06 \mu_{\mathrm{B}}$ in magnetic moment, which might be interpreted as a comparatively

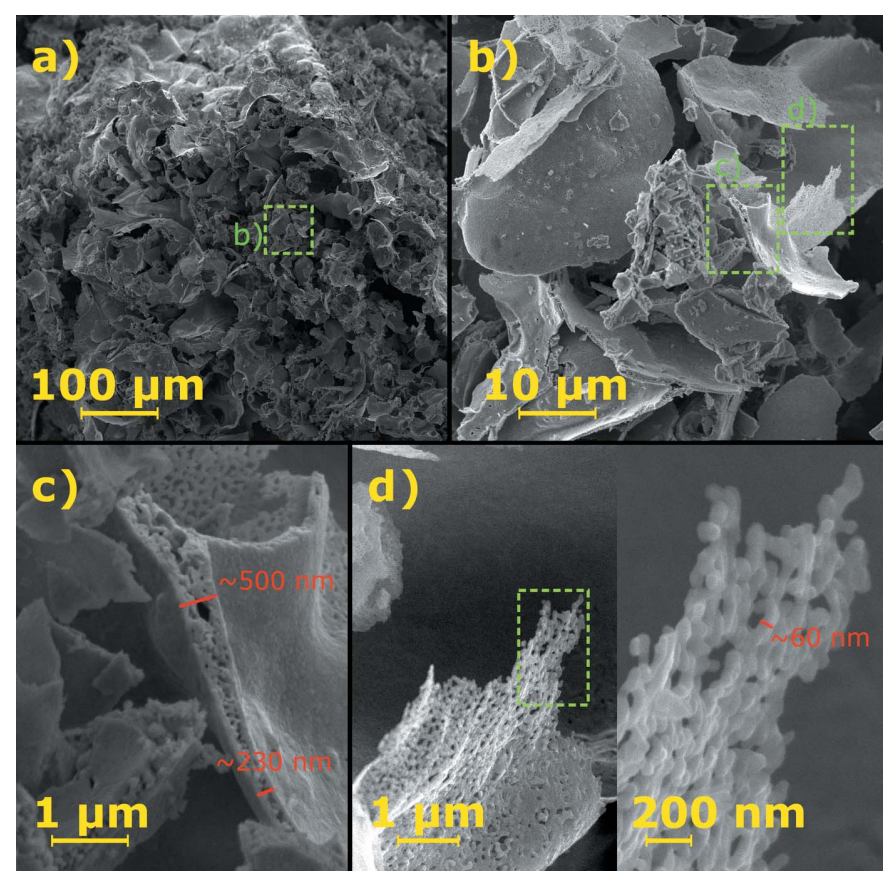

Figure 6

SEM images of the pristine La0.9 sample with different magnifications. A foam-like morphology and globular cavities can be seen in image $(a)$. The structure formed during synthesis and was later crushed into smaller pieces during the grinding step. In image $(b)$, more details of the crumbled pieces are visible at higher magnification. Image $(c)$ displays a section of a cavity wall. While there are pores inside, the outside parts are denser. The thickness ranges from $\sim 230$ to $500 \mathrm{~nm}$. Very porous structures exist as well, as is shown in image $(d)$ (a higher accelerating voltage of $20 \mathrm{kV}$ was used for increased resolution). They are built up by crystallites of sizes around $60 \mathrm{~nm}$, grown together and forming a network. miniscule partial oxidation. This is further supported by a minor contribution of spin-down $\mathrm{Fe}^{3+} d$ states to the empty states above the Fermi level.

$\mathrm{Nd}_{0.875} \mathrm{Ca}_{0.125} \mathrm{FeO}_{3}$ [see Fig. S6 (top) in the supporting information] behaves similarly to $\mathrm{La}_{0.875} \mathrm{Ca}_{0.125} \mathrm{FeO}_{3}$, with the electron hole delocalized over many $\mathrm{O}$ atoms. However, slight changes of the magnetic moments (around $0.1 \mu_{\mathrm{B}}$ ) indicate additional partial oxidation of spin-up Fe atoms, which is not present in the $\mathrm{La}$ analogue. As shown previously by the authors (Lindenthal et al., 2020), $\mathrm{Nd}_{0.5} \mathrm{Ca}_{0.5} \mathrm{FeO}_{3}$ [see Fig. S6 (bottom) in the supporting information] shows empty O- $p$ and $\mathrm{Fe}^{4+}-d$ states above the Fermi level, comparable to $\mathrm{La}_{0.5} \mathrm{Ca}_{0.5}$ $\mathrm{FeO}_{3}$ (with slightly larger changes in the $\mathrm{Fe}$ moments of $0.6 \mu_{\mathrm{B}}$ ). However, for this material, the $\mathrm{Fe}^{4+}$ moments of both spins change by the same amount, while the $\mathrm{Fe}^{3+}$ moments do not change at all compared to the bulk.

Fig. 5 shows changes in the calculated $\mathrm{Fe}-\mathrm{O}$ distances in the perovskites containing $50 \% \mathrm{Ca}$ at the $A$-site due to doping. Both bulk $\mathrm{LaFeO}_{3}$ (Fig. 5a) and bulk $\mathrm{NdFeO}_{3}$ (Fig. 5c) exhibit virtually undistorted $\mathrm{Fe}-\mathrm{O}$ octahedra (the difference between the shortest and longest distances is of the order of $1 \%$ ), while a clear tilting of the octahedra can be seen. In addition to the tilting, the doped perovskites $\left[\mathrm{La}_{0.5} \mathrm{Ca}_{0.5} \mathrm{FeO}_{3}\right.$ in Fig. $5 b$ and $\mathrm{Nd}_{0.5} \mathrm{Ca}_{0.5} \mathrm{FeO}_{3}$ in Fig. $\left.5 d\right]$ show distortion compared to the bulk distances. In both cases, two of the four $\mathrm{Fe}$ atoms of the unit cell (shaded blue and marked $\left(\mathrm{Fe}^{4+}\right.$ ) display a pronounced Jahn-Teller distortion, where $\mathrm{Fe}-\mathrm{O}$ distances along two axes of the octahedra contract by about $5-7 \%$, while the bond along the third axis elongates by $3-7 \%$, which serves as a strong indication of a change of the oxidation state to $\mathrm{Fe}^{4+}$. The magnetic moment of these $\mathrm{Fe}$ atoms are significantly reduced (see above), further hinting toward a partial oxidation. The second type of Fe atoms (shaded yellow and marked ' $\mathrm{Fe}^{3+}$ ) behave differently in the two materials. In $\mathrm{Nd}_{0.5} \mathrm{Ca}_{0.5} \mathrm{FeO}_{3}$, the distances along two axes remain unchanged compared to the bulk, while one distance gets elongated by about $5 \%$. In $\mathrm{La}_{0.5} \mathrm{Ca}_{0.5} \mathrm{FeO}_{3}$, one $\mathrm{Fe}$ atom [marked with an asterisk (*) in Fig. $5 b$ ] behaves similarly to the $\mathrm{Nd}$ analogue, aside from a larger elongation, while for the second atom [marked with a double asterisk (**) in Fig. 5b], two bonds lengthen by 3 and $5 \%$, respectively, while the third remains unchanged. This - together with the slightly changed magnetic moment of this atom - might be a further hint toward a very slight partial oxidation.

In the case of a smaller doping concentration (12.5\%), no pronounced Jahn-Teller effect is found for either $\mathrm{La}$ or $\mathrm{Nd}$ perovskites. Here, the distortion most likely arises primarily due to size effects of the dopant.

\subsection{Macroscopic structure of the doped perovskites}

This section focuses on the morphological characterization of the pristine perovskite powders by SEM. As examples, the La0.9 and NdCo samples are shown here, with further images and other materials shown in the supporting information (Figs. S7-S9). 
Fig. 6 shows images of the La0.9 sample. The powder consists of smaller crumbled pieces, some of them resembling globular cavities (Figs. $6 a$ and $6 b$ ). These result from the synthesis, where the emerging gases (from combustion of the organic species) were responsible for the formation of a foamlike structure of the perovskite material during heating of the gel and calcination. Similar macroscopic structures after synthesis were observed for $\mathrm{LaFeO}_{3}$ by the group of Biniwale (Gosavi \& Biniwale, 2010). Later, when the material was ground, the foam cavities were crumbled but retained their globular microstructure (one of the cavities is shown in more detail in Fig. S7 in the supporting information). A cross section of a cavity wall at high magnification (Fig. $6 c$ ) reveals that its thickness ranges between 230 and $500 \mathrm{~nm}$. Furthermore, only the outside parts of the wall are dense, with the inside being quite porous. Besides the cavity walls, there exist very porous structures, probably grown inside the bubbles (Fig. $6 d$ ), consisting of networks of crystallites with sizes around $60 \mathrm{~nm}$.

Although there are generally slight differences between the different examined materials, the other synthesized perovskites exhibit a very similar morphology compared to La0.9. The same crumbled pieces of the foam structure are found for La0.6 (Fig. S8), Nd0.9 (Fig. S9) and NdCo (Fig. 7). Also, the cavity walls in the undoped materials resemble the morphology of those in La0.9, with denser outermost layers built from distinguishable crystallites, a porous inner structure and similar thicknesses. On the other hand, the Co-doped perovskite material behaves slightly differently. While the inside of the cavity wall of the NdCo sample, as shown in Fig. $7 b$, is as porous as in the other samples, its outermost layer looks different. It is still denser than the inside and very smooth with hardly distinguishable crystallites. Unlike the samples without doping, several pores with diameters up to $70 \mathrm{~nm}$ reach the surface. The wall thickness at this part of the sample varies widely. Parts with a similar thickness $(400 \mathrm{~nm})$ as in La0.9, but also with very thin walls (around $150 \mathrm{~nm}$ thick)

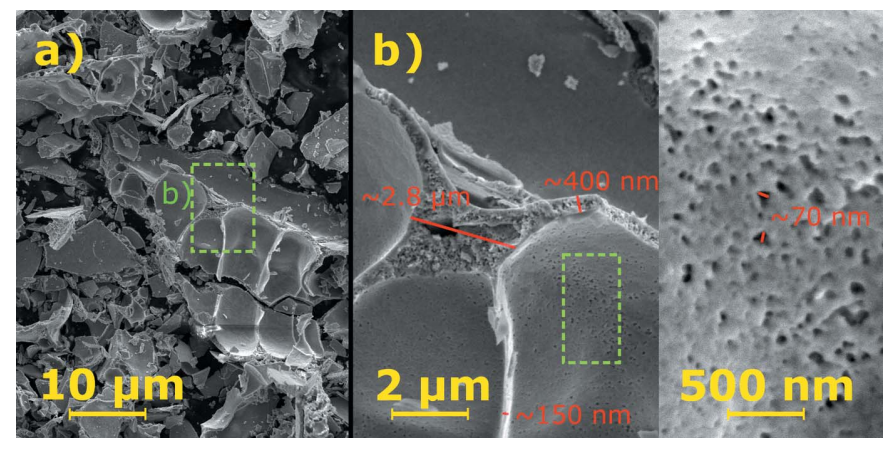

Figure 7

SEM images of the pristine NdCo sample with different magnifications. In image $(a)$, the foam-like morphology is clearly visible. Parts of the structure show connected cavities. The morphology of the wall is observable in image $(b)$. Similar to the other samples, it is porous inside, while the outside layers are denser. However, several pores reach up to the surface and are visible as holes with a diameter of around $70 \mathrm{~nm}$. Unlike in the other samples, the crystallites building up the walls are not distinguishable. The wall thickness varies from thin walls of $\sim 150 \mathrm{~nm}$ to very thick parts of $\sim 2.8 \mu \mathrm{m}$ at a crossing of several cavities. and very thick parts (around $2.8 \mu \mathrm{m}$ thick), where the walls of several cavities meet, can be observed.

A possible explanation for the slightly changed surface morphology in $\mathrm{NdCo}$ is that more pronounced sintering occurred in the calcination step, resulting in the smoothly fused crystallites. At the same time, the holes where the emerging gases left the structure were not clustered around bumps (as they were in La0.9; Fig. S7), but were distributed more uniformly over the whole surface. Similar observations have been made in a study on the grain growth and porosity of perovskites by Tan et al. (2003), where sintering resulted in denser structures.

\subsection{Characterization of exsolution properties}

To investigate the behaviour of the doped perovskites under reducing conditions and to determine if exsolution of $\mathrm{Fe}$ and/or Co can be achieved, in situ XRD studies were performed. For these, the samples were mounted in the reaction chamber of a diffractometer in Bragg-Brentano $\theta-\theta$ geometry. Each experiment started with a pretreatment in $\mathrm{O}_{2}$ at $600{ }^{\circ} \mathrm{C}$ for $30 \mathrm{~min}$. This was done in order to always have a fully oxidized material with the least possible amounts of oxygen vacancies, and thus a defined starting point. After cooling to room temperature, the atmosphere was changed to reducing conditions. For this, $\mathrm{H}_{2}$ was guided through a bubbler filled with water at atmospheric pressure. In this way, a ratio of $\mathrm{H}_{2}$ to $\mathrm{H}_{2} \mathrm{O}$ of around 32:1 was obtained for the humidified $\mathrm{H}_{2}$, which corresponds to an equivalent oxygen partial pressure $p\left(\mathrm{O}_{2}\right)$ of only $5.3 \times 10^{-26}$ bar (for details, see $\S \mathrm{S} 6$ in the supporting information). The role of water in the gas phase is to provide an oxygen source for a well-defined $p\left(\mathrm{O}_{2}\right)$. This is required to be able to compare exactly our results with future electrochemical studies on these materials and with other work on exsolution in the literature (Opitz et al., 2017).

Experiments were conducted with a constant gas flow of around $0.51 \mathrm{~min}^{-1}$, the temperature was increased stepwise

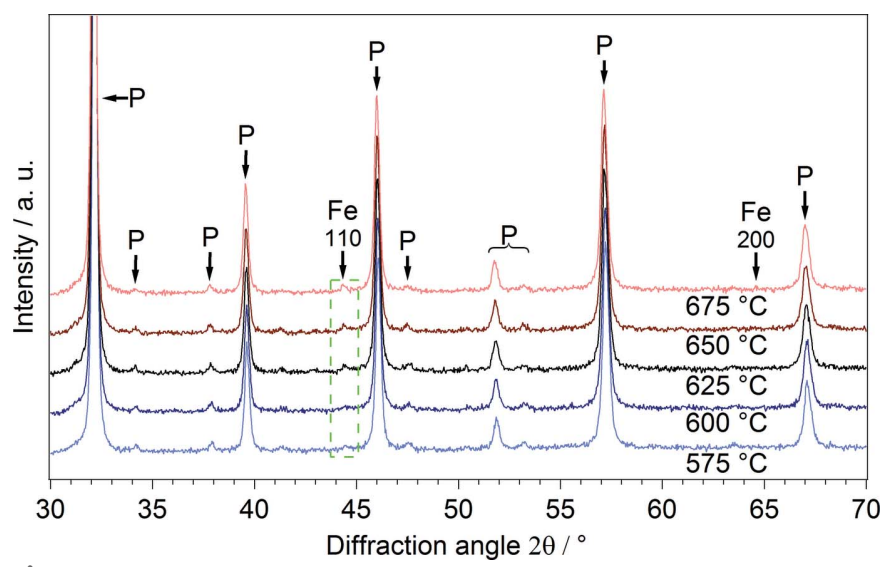

Figure 8

Diffractograms of the La0.9 sample during the reduction with wet $\mathrm{H}_{2}$ at increasing temperature. The perovskite structure (peaks labelled 'P'; for the respective $h k l$ indices, $c f$. Fig. 2) stayed intact over the whole temperature range and no $\mathrm{La}_{2} \mathrm{O}_{3}$ phase was formed. A small peak of elemental $\mathrm{Fe}$ appeared (green border), first clearly seen at $625^{\circ} \mathrm{C}$, indicating $\mathrm{Fe}$ nanoparticle exsolution. 
and at each step an XRD measurement was taken after waiting for $30 \mathrm{~min}$. The reflections of the new phases in the diffractograms were assigned according to database entries in the ICDD PDF-4+ 2019 database (ICDD, 2018) and are, in the following figures, labelled with the respective $h k l$ indices. The reflections of the perovskite phase are labelled with 'P'; for their $h \mathrm{kl}$ indices, refer to Fig. 2. The results of in situ reduction of La0.9 can be seen in Fig. 8. The appearance of a diffraction peak at $2 \theta$ of $44.4^{\circ}$ was observed, which could be assigned to metallic $\mathrm{Fe}$, thus indicating that exsolution occurred, i.e. formation of metal (nano)particles on the surface of the perovskite. It is first clearly distinguishable at $625^{\circ} \mathrm{C}$. Furthermore, no decomposition of the perovskite (formation of an $\mathrm{La}_{2} \mathrm{O}_{3}$ phase) took place over the whole temperature range tested (up to $675^{\circ} \mathrm{C}$ ).

During the in situ reduction of La0.6 with humidified $\mathrm{H}_{2}$, the diffraction peak corresponding to metallic $\mathrm{Fe}\left(2 \theta\right.$ of $\left.44.4^{\circ}\right)$ started to appear at slightly higher temperatures $\left(650^{\circ} \mathrm{C}\right)$ than for La0.9 (Fig. S10), but generally showing the same trend as observed before with less Ca doping. Up to $675^{\circ} \mathrm{C}$, there was no decomposition of the material and no formation of $\mathrm{CaO}$ was observed, although the Ca content of this material was much higher.

To prove that exsolution actually occurred, SEM measurements were performed after reduction of La0.6 in wet $\mathrm{H}_{2}$ (Fig. 9). The sample used for the in situ XRD experiment was investigated directly after the last temperature step at $675^{\circ} \mathrm{C}$ (Fig. 9b). Additionally, another batch of the pristine sample was freshly reduced in a flow reactor at $650{ }^{\circ} \mathrm{C}$, i.e. the temperature at which exsolution was first observed in the in

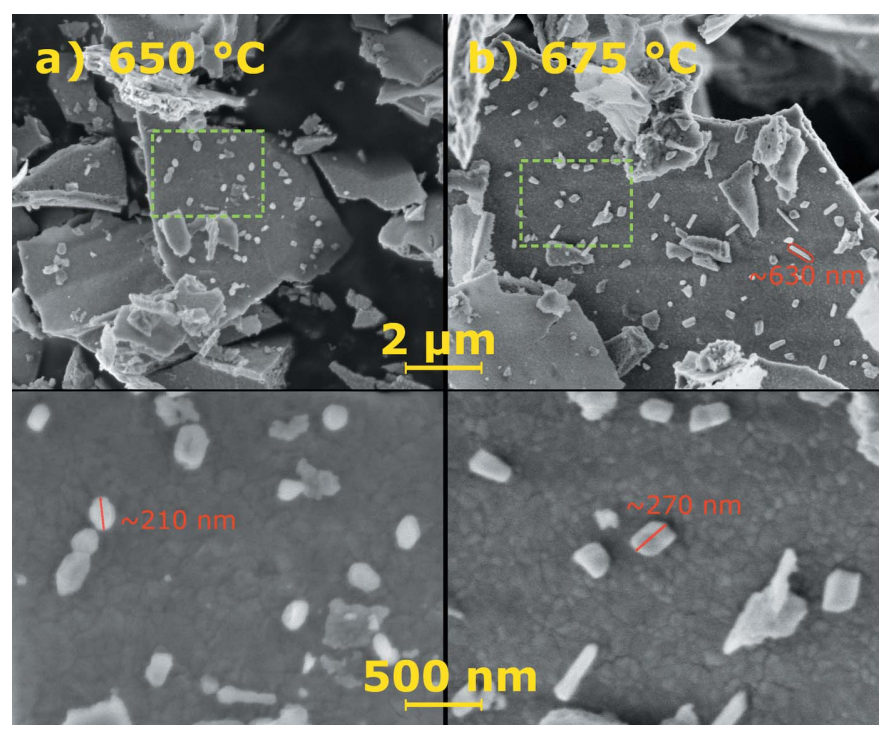

Figure 9

SEM images of the La0.6 sample after reduction in humid $\mathrm{H}_{2}$ at $(a) 650$ and $(b) 675^{\circ} \mathrm{C}$. The lower images show details of the images in the first row at a higher magnification (green box). Exsolved nanoparticles decorate the perovskite surface. At $650^{\circ} \mathrm{C}$, they are smaller, with diameters $\sim 210 \mathrm{~nm}$. Some are very regularly shaped, indicating a controlled crystal growth. On the other hand, at $675^{\circ} \mathrm{C}$, the nanoparticles are larger on average. While some retain the regular shape, with diameters of $\sim 270 \mathrm{~nm}$, a needle-like growth set in. The longest needles are around $630 \mathrm{~nm}$ in length. situ XRD experiment (Fig. 9a). In both samples, nanoparticles can be seen decorating the perovskite surface. After reduction at $650{ }^{\circ} \mathrm{C}$, they are regularly shaped, some of them with the geometries of smoothly grown crystals, distinguishing them from crumbled pieces of the perovskite material after grinding. They have diameters of around $210 \mathrm{~nm}$. After reduction at $675^{\circ} \mathrm{C}$, the particles are larger on average, with more variation in shape. Some still have quite regular shapes, with diameters around $270 \mathrm{~nm}$, but also needle-like particles can be observed. The largest needles have lengths of around $630 \mathrm{~nm}$. The higher temperature facilitated further growth of the nanoparticles after their formation, partly needle-like, but no obvious difference in the number of formed particles was found. A needle-like Fe exsolution from LSF has already been reported by Thalinger et al. (2015).

One particle of the La0.6 sample after reduction at up to $675^{\circ} \mathrm{C}$ was further investigated with EDX. A line scan was performed across the exsolved particle. The net intensities of the Fe $L$, the $\mathrm{O} K$ and the La $M$ peaks along the line are displayed in Fig. 10. At the position of the particle, the Fe peak increases significantly, while at the same time the $\mathrm{O}$ peak (strongly) and the La peak (slightly) decrease. This confirms the assumption of Fe nanoparticle exsolution, and that the $\mathrm{Fe}$ phase observed in the diffractograms can indeed be attributed to the nanoparticles visible with SEM. These findings prove that exsolution of $\mathrm{Fe}$ nanoparticles upon reduction was successful.

The diameter of the particle in the direction of the scan can be determined, considering that, due to the limited resolution of EDX, the Fe signal increase is not a step, but follows a slope. As the signal for one pixel originated from a nonzero

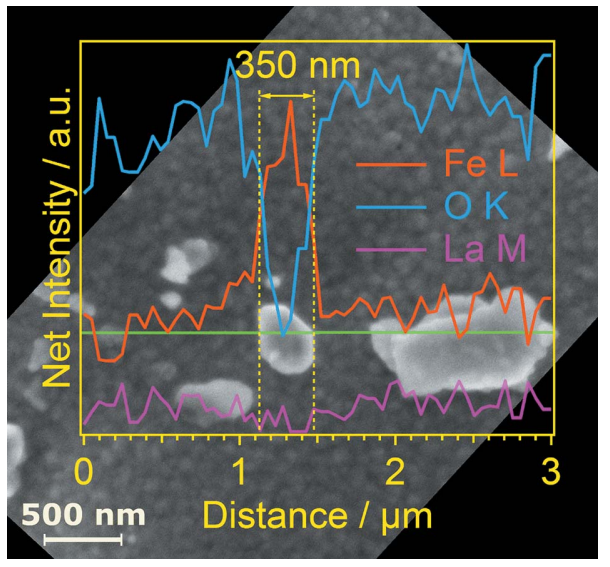

Figure 10

SEM secondary electron image of the La0.6 sample after reduction in wet $\mathrm{H}_{2}$ at $675^{\circ} \mathrm{C}$. A regularly shaped particle is visible in the centre. An EDX scan was performed along the green line crossing the particle. The overlaid diagram shows the net intensity of $\mathrm{Fe}, \mathrm{O}$ and $\mathrm{La}$ peaks in the recorded EDX spectra. When crossing the particle in the centre of the image, the Fe $L$ peak increases, while the $\mathrm{O} K$ peak and the La $M$ peak decrease. The positions of the extremal slopes of the Fe signal are marked with yellow lines. These positions coincide with the edges of the particle in the secondary electron image. The particle diameter in the direction of the line is $350 \mathrm{~nm}$. In contrast, the signals do not change when crossing the particle on the right, which is just a crumbled piece of the perovskite material. 
area, the extent of the signal increase reflects the percentage of the signal originating from the particle. When the beam was at the position of the centre of the particle, the Fe peak reached its maximum value. The edges of the particle are assumed to be located at the position where the slope of the $\mathrm{Fe}$ signal has the maximum value (which means a maximum change of $\mathrm{Fe}$ content). Thus, a diameter of $350 \mathrm{~nm}$ was determined, agreeing well with the diameter obtained from the secondary electron image.

The line scan also crosses another particle, but without a significant change in any of the signals. This particle is probably a finely crumbled piece of the perovskite material. It also lacks a regular morphology, while the exsolved and grown $\mathrm{Fe}$ particle is smoothly shaped and shows some symmetry (the projection resembles a polygon with six corners and parallel edges).

For the Nd-based $A$-site-doped materials, in situ XRD experiments were performed in the same way in humidified $\mathrm{H}_{2}$. Exsolution ( $\mathrm{Fe}$ peak at $2 \theta$ of $44.4^{\circ}$ ) from an intact perovskite structure was seen for both $\mathrm{Nd} 0.9$ (Fig. 11) and $\mathrm{Nd} 0.6$ (Fig. S11) at 650 and $675{ }^{\circ} \mathrm{C}$, respectively. This increase of the onset of exsolution upon exchanging $\mathrm{La}$ with $\mathrm{Nd}$ confirms the expected increase of the material stability, which was stated in the Introduction. Please note that owing to an instrumentation problem during the experiment on $\mathrm{Nd} 0.9$, the temperature was erroneously held for $5 \mathrm{~h}$ (instead of $30 \mathrm{~min}$ ) at $650{ }^{\circ} \mathrm{C}$ before the diffractogram was recorded. Thus, the $\mathrm{Fe}$ peak had already clearly evolved. In contrast, for $\mathrm{Nd} 0.6$, as for most other samples, it was only very small at the first temperature where it appeared. This suggests that, in addition to temperature, the reduction time is quite important for the size of the resulting nanoparticles. For both materials, up to the highest temperatures investigated, the perovskite structure was stable and none of the decomposition products $\left(\mathrm{Nd}_{2} \mathrm{O}_{3}\right.$ or $\left.\mathrm{CaO}\right)$ could be observed.

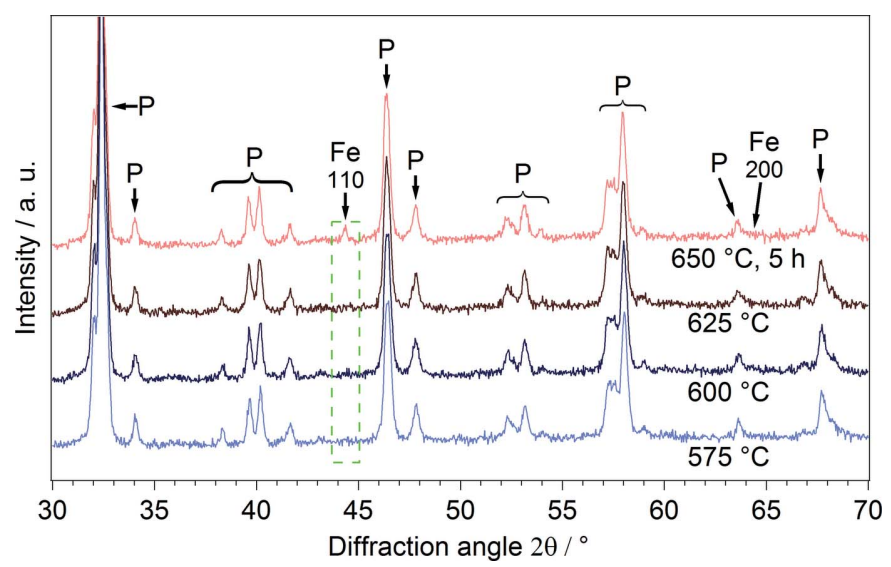

Figure 11

Diffractograms of the Nd0.9 sample during the reduction with wet $\mathrm{H}_{2}$ at increasing temperatures. The material was completely stable up to a temperature of $625^{\circ} \mathrm{C}$. However, after holding the temperature at $650{ }^{\circ} \mathrm{C}$ for $5 \mathrm{~h}$, a peak (green border) corresponding to an elemental Fe phase could be observed, while the perovskite structure (peaks labelled 'P'; for the respective $h k l$ indices, $c f$. Fig. 2) remained intact.
For the investigation with SEM, the Nd0.9 sample was again freshly reduced in a flow reactor in humidified $\mathrm{H}_{2}$ at $650{ }^{\circ} \mathrm{C}$ (Fig. 12). The sample surface is observed at an angle. Thus, it can be clearly seen that the particles were sticking out from the surface. They had diameters of approximately $200 \mathrm{~nm}$. To monitor variations in the composition, an EDX mapping was performed in the region marked with the green box. The net intensity of the $\mathrm{Fe} L$ spectral peak was used for characterizing the Fe distribution in the material, shown as an overlay of the secondary electron image. The positions of the particles coincided well with an increased Fe signal (and a lower $\mathrm{Nd} M$ peak, which is not shown here). Similar to La0.6 (see Fig. 10), this strongly suggests the $\mathrm{Fe}$ nature of the formed particles.

In the in situ XRD experiment with the doped perovskite $\mathrm{NdCo}$ (Fig. 13), already at a temperature of $550{ }^{\circ} \mathrm{C}$, a new Bragg peak arose at a $2 \theta$ value of $44.8^{\circ}$. The peak grew with increasing temperature and shifted to lower angles. At $650{ }^{\circ} \mathrm{C}$, its maximum was at a $2 \theta$ value of $44.5^{\circ}$. In contrast to the Cofree materials, the reflection was significantly more intense, indicating a larger amount of the formed phase. Similar to the other samples, this phase could be ascribed to exsolution of a metallic bcc phase, but it was not clearly distinguishable whether it was a pure phase or an $\mathrm{Fe}-\mathrm{Co}$ alloy. Since exsolution did not occur on the materials without Co doping at such low temperatures (compare with $\mathrm{Nd} 0.6$ with an exsolution onset at $675{ }^{\circ} \mathrm{C}$ ) and since cobalt oxides are generally easier to reduce than iron oxides, it is rather likely that, especially at lower temperatures, the phase contained predominately Co. This assumption is also supported by the position of the bcc (110) reflection at a significantly higher $2 \theta$ value than in the samples without doping $\left(44.8^{\circ}\right.$ at $550{ }^{\circ} \mathrm{C}$ for $\mathrm{NdCo}$ versus $44.4^{\circ}$ at $650{ }^{\circ} \mathrm{C}$ for $\mathrm{Nd} 0.9$ and $\mathrm{Nd} 0.6$ ). It is worth mentioning that the temperature difference is not sufficient to explain this difference (changes of $c a 0.05^{\circ} / 373{ }^{\circ} \mathrm{C}$ are reported for both Co and $\mathrm{Fe}$ in the respective temperature range). Rather, the position of the (110) reflection for pure $\mathrm{Fe}$ and Co can be found in the literature to differ by $0.40 \pm 0.05^{\circ}$.

Also, the angle shift to $44.5^{\circ}$ at $650^{\circ} \mathrm{C}$ (which is again more pronounced than would be expected only due to thermal expansion) could be explained by an increase of the $\mathrm{Fe}$

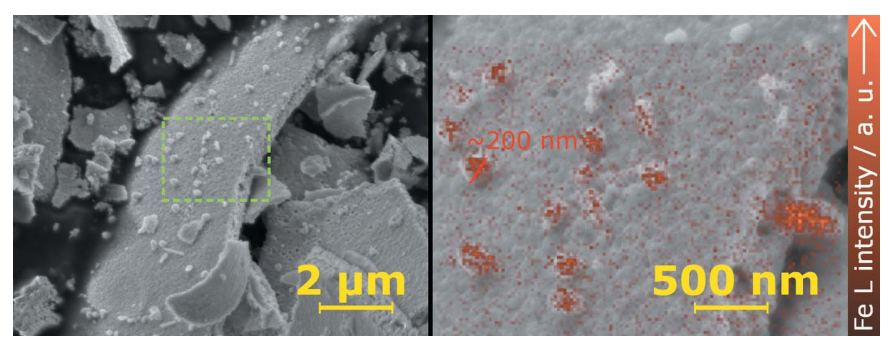

Figure 12

SEM images of the Nd0.9 sample after reduction in wet $\mathrm{H}_{2}$ at $650^{\circ} \mathrm{C}$. The image on the right is a detail (green box) of the image on the left at a higher magnification. Exsolved nanoparticles are visible at the perovskite surface, with diameters of around $200 \mathrm{~nm}$. An EDX mapping was performed of the magnified area. The Fe distribution, characterized by the net intensity of the Fe $L$ peak, is shown as an overlay. The particles visible in the secondary electron image coincided with a higher $\mathrm{Fe}$ concentration. 
content in the exsolved metal particles with increasing temperature. At higher temperatures, when Fe could also be reduced, the composition of the particles would shift toward an $\mathrm{Fe}$-enriched alloy. Because $\mathrm{Fe}^{0}$ has a larger atomic radius than $\mathrm{Co}^{\circ}$, an increase of the Fe content in an Fe-Co alloy is expected to cause an increase of the unit-cell dimensions and thus a decrease of the diffraction angle ( $c f$. Bragg's law). In a related study, Chen et al. (2018) recently reported exsolved $\mathrm{Co}_{1-x} \mathrm{Fe}_{x}$ particles from $\mathrm{La}_{0.5} \mathrm{Sr}_{0.5} \mathrm{Co}_{0.45} \mathrm{Fe}_{0.45} \mathrm{Nb}_{0.1} \mathrm{O}_{3-\delta}$, with an increasing $\mathrm{Fe}$ content at higher temperatures, in accordance with the conclusions reported here.

During the in situ reduction, the perovskite structure remained intact over the whole temperature range tested, but a $\mathrm{CaO}$ phase (peaks at $2 \theta$ values of 37.2 and $53.5^{\circ}$ ) evolved, starting from $600{ }^{\circ} \mathrm{C}$. Interestingly, the appearance of this phase correlates with the onset of the bcc (110) shift, which was interpreted above to be caused by (enhanced) Fe exsolution. This segregation of $\mathrm{CaO}$ may thus be triggered by the $A$-site excess stoichiometry in the perovskite resulting from proceeding exsolution of $B$-site cations (predominantly Fe). A similar segregation of an alkaline earth metal in a perovskite material has already been reported for Sr in LSF (Koo et al., 2018) and for alkaline-earth-metal-doped lanthanum manganite (Kim et al., 2020). However, there is a temperature window between 550 and $575^{\circ} \mathrm{C}$ where exsolution occurs without $\mathrm{CaO}$ segregation. These would be the ideal conditions for nanoparticle exsolution and for further catalytic applications.

Additionally, $\mathrm{NdCo}$ was freshly reduced in a flow reactor in humidified $\mathrm{H}_{2}$ before it was investigated with SEM to prove that exsolution was successful (Fig. 14). A temperature of $575^{\circ} \mathrm{C}$ was chosen for the reduction, to ensure being in the

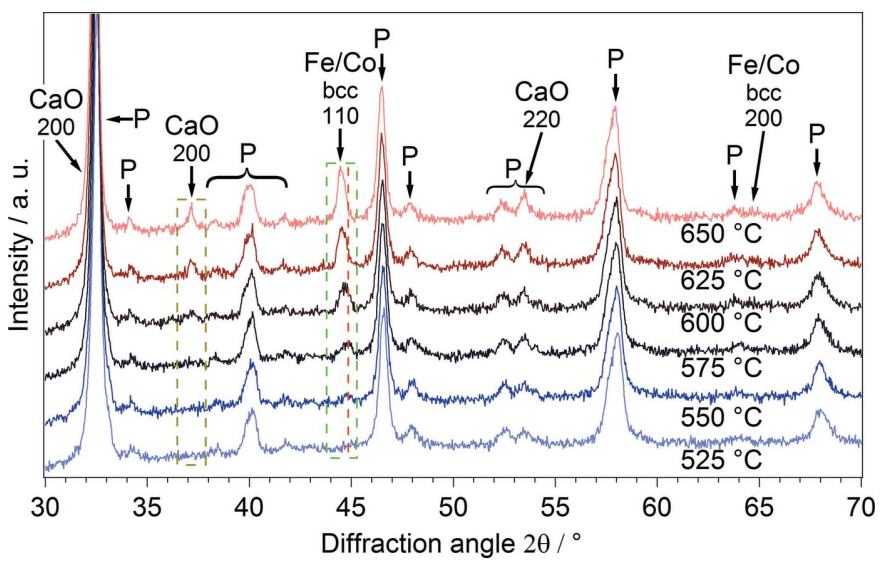

Figure 13

Diffractograms of the NdCo sample during the reduction with humid $\mathrm{H}_{2}$ at increasing temperature. A new phase, which could be assigned to bcc $\mathrm{Fe} / \mathrm{Co}$, was formed above $550{ }^{\circ} \mathrm{C}-$ the corresponding (110) reflection is marked by the green box. A shift of the corresponding peak to lower angles was observed at higher temperatures, which was stronger than expected from thermal expansion (compare position to red line). At $600{ }^{\circ} \mathrm{C}$, a $\mathrm{CaO}$ phase (brown box) appeared. Both the metallic bcc phase and the $\mathrm{CaO}$ phase were more prominent at higher temperatures, while the perovskite structure (reflections labelled 'P'; for the respective $h \mathrm{kl}$ indices, $c f$. Fig. 2) stayed intact.
Table 5

Temperature at which metal exsolution was first observed during the in situ XRD reduction experiments with wet $\mathrm{H}_{2}$.

The temperature is higher for samples with La than with $\mathrm{Nd}$ and higher with a greater amount of doping with Ca. Doping with Co reduces the exsolution temperature by $125^{\circ} \mathrm{C}$. LSF has a much lower exsolution temperature than the Ca-doped samples. Doping with $\mathrm{Ca}$ instead of $\mathrm{Sr}$ increases the exsolution temperature by $225^{\circ} \mathrm{C}$. In addition, the Glazer angles (see \$3.2) of the respective materials are given.

\begin{tabular}{llcc}
\hline & & \multicolumn{2}{c}{ Glazer angles } \\
\cline { 3 - 4 } Sample & Exsolution temperature $\left({ }^{\circ} \mathrm{C}\right)$ & $a\left(^{\circ}\right)$ & $b\left({ }^{\circ}\right)$ \\
\hline La0.9 & 625 & 7.47 & 8.69 \\
La0.6 & 650 & 8.16 & 8.66 \\
Nd0.9 & 650 & 10.18 & 8.73 \\
Nd0.6 & 675 & 10.84 & 6.22 \\
NdCo & 550 & 10.84 & 6.22 \\
LSF & 425 & 4.84 & - \\
\hline
\end{tabular}

ideal temperature window for exsolution without $\mathrm{CaO}$ segregation. Very small and homogeneously distributed nanoparticles are visible. They are regularly shaped, with diameters around $50 \mathrm{~nm}$.

As a comparison, commercially available LSF was also tested for exsolution (see Figs. S12 and S13 in the supporting information). Here, exsolution of Fe (Bragg peak at $44.5^{\circ}$ ) had started already at $425^{\circ} \mathrm{C}$. The perovskite structure was intact and no segregation of an Sr-containing phase could be observed over the whole temperature range (up to $500{ }^{\circ} \mathrm{C}$ ).

\subsection{Comparison of perovskite materials}

In Table 5, the materials are compared with respect to the lowest temperature at which exsolution occurred during the in situ XRD experiments. This value reflects the stability of the respective materials and how easily exsolution can be achieved.

Several trends can be observed. Using $\mathrm{Nd}$ on the $A$-site instead of $\mathrm{La}$ with the same amount of $\mathrm{Ca}$ doping slightly increased the exsolution temperature by $25^{\circ} \mathrm{C}$ (from 625 to $650{ }^{\circ} \mathrm{C}$ for $\mathrm{La0} .9$ and $\mathrm{Nd} 0.9$, and from 650 to $675^{\circ} \mathrm{C}$ for $\mathrm{La} 0.6$

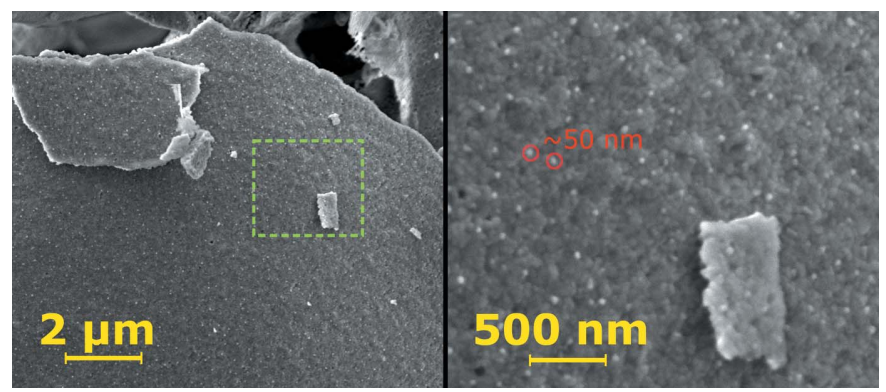

Figure 14

SEM images after reduction in humidified $\mathrm{H}_{2}$ of the NdCo sample at $575^{\circ} \mathrm{C}$. The image on the right is a detail of the image on the left (green box) at higher magnification. Uniformly distributed exsolved nanoparticles up to $50 \mathrm{~nm}$ in size decorated the perovskite surface. There were more particles and they were smaller than for Nd0.9 (diameters around $200 \mathrm{~nm}$ ). Similar to Fig. 10, there is a crumbled piece of the perovskite visible in the lower right corner of the magnified area. 
and Nd0.6). As La and $\mathrm{Nd}$ are chemically similar, an explanation could be based on the crystal structure of the materials. Due to their size, the La ions fit better into the perovskite structure, whereas the smaller $\mathrm{Nd}$ ions lead to more pronounced distortions. This is reflected in larger values for the Glazer angles (most relevant for this consideration is angle $a$, as angle $b$ only changes in the case of $\mathrm{Nd} 0.6$ ) of the $\mathrm{Fe}$ coordination octahedra (see Fig. 3 and Table 4) of the samples with $\mathrm{Nd}$ compared to those with $\mathrm{La}$. A less distorted structure seems to facilitate the exsolution process, but it is not yet clear if that happened due to thermodynamics (less stable perovskite structure) or because of a kinetic effect (easier $B$-metal migration through a structure with fewer distortions).

Similarly, exchanging $\mathrm{Ca}$ with $\mathrm{Sr}$ (while leaving the amount of doping constant) resulted in an exsolution temperature of $425^{\circ} \mathrm{C}$ for LSF (see supporting information), a significant decrease by $225^{\circ} \mathrm{C}$ with respect to La0.6 $\left(650{ }^{\circ} \mathrm{C}\right)$. In this case, the change of structure accompanying the change of the $A$-site element even lead to a different symmetry (orthorhombic for La0.6 versus rhombohedral for LSF). The rhombohedral structure deviates less from the ideal cubic perovskite structure. In accordance with the proposed effect of easier exsolution from a less distorted structure, this symmetry change could explain the large temperature difference between $\mathrm{Ca}$ and $\mathrm{Sr}$ doping. Another possible factor is the crystallinity and morphology of the sample, which is different for the commercial LSF material than for the freshly prepared perovskite materials.

The results seem to contradict those of other groups who found the opposing trend that higher symmetry increases the exsolution temperature (Steiger et al., 2019) and larger distortions due to strain enhance exsolution (Han et al., 2019). Therefore, further experiments are necessary to fully understand the observed behaviour.

The change of the amount of $\mathrm{Ca}$ doping also changed the distortion. In the case of $\mathrm{La} 0.9$ and $\mathrm{La} 0.6$, a greater amount of $\mathrm{Ca}$ increased the distortion. As before, this increased the exsolution temperature from $625^{\circ} \mathrm{C}$ for $\mathrm{La} 0.9$ to $650^{\circ} \mathrm{C}$ for La0.6. However, a contrary trend was observed for $\mathrm{Nd} 0.9$ and $\mathrm{Nd} 0.6$. Here, the higher $\mathrm{Ca}$ content slightly decreased the distortion (the larger Glazer angle $a$ is compensated by the smaller angle $b$ ). Nevertheless, the exsolution temperature was still higher for $\mathrm{Nd} 0.6\left(675^{\circ} \mathrm{C}\right)$ than for $\mathrm{Nd} 0.9\left(650^{\circ} \mathrm{C}\right)$. Also for $\mathrm{La} 0.9$ and La0.6, the distortions were similar and the difference was probably not big enough to explain the increase of exsolution temperature alone. Apparently, the changed electronic properties (see \$3.3) may play a role here, but again the effect is not yet understood.

Another observation was that $B$-site doping with Co reduces the exsolution temperature. This metal is more easily reducible than $\mathrm{Fe}$. Thus, the required temperature was lower than for $\mathrm{Nd} 0.6$, which had the same $A$-site composition $\left(550{ }^{\circ} \mathrm{C}\right.$ for $\mathrm{NdCo}$ versus $675^{\circ} \mathrm{C}$ for $\left.\mathrm{Nd} 0.6\right)$. Jiang et al. (2020) found exsolution of a Co-Fe alloy from $\mathrm{La}_{0.9} \mathrm{Fe}_{0.9} \mathrm{Co}_{0.1} \mathrm{O}_{3-\delta}$ even at $500{ }^{\circ} \mathrm{C}$ after reducing for $4 \mathrm{~h}$. Their study agrees with ours in that exsolution at that temperature is not possible without Co doping. This is also in accordance with theoretical considerations by Kwon et al. (2017) regarding exsolution trends. Furthermore, with Co doping the resulting nanoparticles are smaller (around $40 \mathrm{~nm}$ for $\mathrm{NdCo}$ ) and more homogeneously distributed. The Co-doped perovskite has the lowest exsolution temperature of all synthesized perovskites, which makes it an interesting starting point for further investigations. Similar to the effect of a different amount of $\mathrm{Ca}$ doping, the varying exsolution temperatures for different $B$-site dopings could not be explained by a changed perovskite structure, but by the reducibility of the $B$-site cations. Both parameters, i.e. perovskite structure and $B$-site cation reducibility, defined the resulting exsolution behaviour.

In addition to the exsolution properties, the reduction behaviour of the materials differed by their tendency for $\mathrm{CaO}$ segregation. In general, Kim et al. (2020) showed that segregation of the alkaline earth $A$-site dopant can occur both under oxidizing and reducing conditions. However, in this study, $\mathrm{CaO}$ was only observed in the samples with $\mathrm{Co} B$-site doping during reduction. If $\mathrm{CaO}$ segregation happened with the other materials and/or under oxidizing atmosphere, the amount of $\mathrm{CaO}$ was too low to detect with the methods used. For the Co-doped material, the segregation started at $600{ }^{\circ} \mathrm{C}$, indicating that the perovskite structure was not completely stable anymore and a partial decomposition set in. In this case, the segregation accompanied the formation of an exsolved metallic phase. This leads to the conclusion that $\mathrm{CaO}$ segregation is driven not only by oxygen vacancy formation (as described by Kim et al.) but also by the necessity to balance the stoichiometry of the perovskite structure, as $B$-site cation exsolution otherwise would result in an $A$-site excess, resulting in a sufficiently large and hence detectable amount of $\mathrm{CaO}$. The reason why no $\mathrm{CaO}$ was found for the other samples might be the amount of exsolved metal. This amount was significantly higher for the Co-doped sample, where the better reducibility facilitated the exsolution process. A possibility to reduce $\mathrm{CaO}$ segregation might be the use of $A$-site deficient perovskite materials as a starting point for exsolution. Here, exsolution of $B$-site cations would establish the stoichiometry, thus strengthening the perovskite structure stability instead of decreasing it (Sun et al., 2015).

\section{Conclusions}

The investigated perovskite materials, i.e. $\mathrm{La}_{0.9} \mathrm{Ca}_{0.1} \mathrm{FeO}_{3-\delta}$, $\mathrm{La}_{0.6} \mathrm{Ca}_{0.4} \mathrm{FeO}_{3-\delta}, \quad \mathrm{Nd}_{0.9} \mathrm{Ca}_{0.1} \mathrm{FeO}_{3-\delta}, \quad \mathrm{Nd}_{0.6} \mathrm{Ca}_{0.4} \mathrm{FeO}_{3-\delta}$ and $\mathrm{Nd}_{0.6} \mathrm{Ca}_{0.4} \mathrm{Fe}_{0.9} \mathrm{Co}_{0.1} \mathrm{O}_{3-\delta}$, were successfully synthesized, which was proven by ICP-OES (composition) and XRD (structure) measurements. Their crystallographic structures were determined by Rietveld refinements of the XRD pattern and the results were supported by DFT calculations.

The structures are isotypic and can be derived from the ideal cubic perovskite structure. The average $A$-site ion radius is too small for a cubic perovskite structure, which results in tilting of the $\mathrm{Fe}$ coordination octahedra according to the Glazer system $a^{-} b^{+} a^{-}$. This reduces the symmetry and the unit cell becomes orthorhombic. The extent of tilting depends on the mismatch of the ionic radii of the involved cations. 
Exchanging the larger $\mathrm{La}^{3+}$ ion with the smaller $\mathrm{Nd}^{3+}$ ion increases the distortions and reduces the cell volume.

The effect of Ca doping could be understood by means of DFT calculations, which revealed that the addition of $\mathrm{Ca}$ changes the electronic structure of the materials (visible in the DOS). The undoped bulk perovskites are insulating, but due to the introduction of $\mathrm{Ca}^{2+}$ on the $A$-site of the perovskite, electron holes are created as charge compensation. This electron hole is delocalized over many $\mathrm{O}$ atoms ( $p$ states) and Fe atoms ( $d$ states); thus, the $A$-site-doped perovskites show metallic behaviour. For the materials with lower Ca doping, the DOS changes are mainly limited to shifts relative to the Fermi level, while the DOS shape remains qualitatively almost unchanged. In contrast, a partial oxidation of $\mathrm{Fe}$ is found for the perovskites with more $\mathrm{Ca}$. Here, half of the $\mathrm{Fe}$ atoms change their oxidation state from $\mathrm{Fe}^{3+}$ to $\mathrm{Fe}^{4+}$. This is supported by a significant reduction of magnetic moments, accompanied by a Jahn-Teller distortion of their O-atom coordination octahedra, where the $\mathrm{Fe}-\mathrm{O}$ distances along two axes are contracted while the bond along the third axis is elongated.

The exsolution behaviour of the materials was investigated with in situ XRD experiments and SEM. For all of the five examined perovskites, $\mathrm{Fe}$ (or $\mathrm{Fe} / \mathrm{Co}$ in the case of the Codoped material) nanoparticle formation on the surface could be observed upon controlled reduction in a humidified hydrogen atmosphere. The particle sizes mostly ranged from 150 to $350 \mathrm{~nm}$ for the materials without $B$-site doping, while with Co doping the particles were smaller (around $50 \mathrm{~nm}$ ) and more homogeneously distributed. An easier exsolution (already at lower temperatures) was found for less distorted structures and less Ca doping. Doping with the more easily reduced Co strongly facilitated the exsolution process and reduced the necessary temperature for exsolution significantly. The observations suggest a preferential exsolution of Co over Fe for low exsolution temperatures. The easy exsolution of well-dispersed Co nanoparticles makes the material a highly promising candidate as a catalyst for reactions related to chemical energy conversion.

The results show that fine-tuning of the perovskite composition will allow tailored exsolution of nanoparticles. The exsolution process (necessary conditions and resulting exsolved nanoparticles) can be influenced by structural distortions, introduction of $A$-site acceptor dopants and doping with more or less reducible and catalytically active $B$-site transition metals. This knowledge is the basis for a rational material design and can be used to create catalysts with properties well adjusted to their intended application and operation environment.

\section{Acknowledgements}

We want to thank Werner Artner for creative support during the in situ XRD measurements, which were carried out within the X-ray Center of TU Wien. The computational results presented have been achieved using the Vienna Scientific Cluster (VSC).

\section{Funding information}

This research was funded by the European Research Council (ERC) under the European Union's Horizon 2020 research and innovation programme, grant agreement no. 755744/ ERC-Starting Grant TUCAS.

\section{References}

Adijanto, L., Padmanabhan, V. B., Gorte, R. J. \& Vohs, J. M. (2012). J. Electrochem. Soc. 159, F751-F756.

Ahmadi, M., Wu, T. \& Hu, B. (2017). Adv. Mater. 29, 1605242.

Anisimov, V. I., Zaanen, J. \& Andersen, O. K. (1991). Phys. Rev. B, 44, 943-954.

Bartolomé, J., Palacios, E., Kuz'Min, M. D., Bartolomé, F., Sosnowska, I., Przeniosło, R., Sonntag, R. \& Lukina, M. M. (1997). Phys. Rev. $B, \mathbf{5 5}, 11432-11441$.

Blaha, P., Schwarz, K., Madsen, G. K. H., Kvasnicka, D., Luitz, J., Laskowski, R., Tran, F. \& Marks, L. D. (2018). WIEN2k: An Augmented Plave Wave plus Local Orbitals Program for Calculating Crystal Properties. Vienna University of Technology.

Cascos, V., Fernández-Díaz, M. T. \& Alonso, J. A. (2019). Renew. Energy, 133, 205-215.

Chen, X., Ni, W., Wang, J., Zhong, Q., Han, M. \& Zhu, T. (2018). Electrochim. Acta, 277, 226-234.

Degen, T., Sadki, M., Bron, E., König, U. \& Nénert, G. (2014). Powder Diffr. 29, S13-S18.

Eyssler, A., Kleymenov, E., Kupferschmid, A., Nachtegaal, M., Kumar, M. S., Hug, P., Weidenkaff, A. \& Ferri, D. (2011). J. Phys. Chem. C, 115, 1231-1239.

Falcón, H., Goeta, A. E., Punte, G. \& Carbonio, R. E. (1997). J. Solid State Chem. 133, 379-385.

Fossdal, A., Menon, M., Waernhus, I., Wiik, K., Einarsrud, M. A. \& Grande, T. (2004). J. Am. Ceram. Soc. 87, 1952-1958.

Glazer, A. M. (1972). Acta Cryst. B28, 3384-3392.

Goldschmidt, V. M. (1926). Naturwissenschaften, 14, 477-485.

Gorte, R. J. \& Vohs, J. M. (2009). Curr. Opin. Colloid Interface Sci. 14, 236-244.

Gosavi, P. V. \& Biniwale, R. B. (2010). Mater. Chem. Phys. 119, 324 329.

Haag, J. M., Barnett, S. A., Richardson, J. W. \& Poeppelmeier, K. R. (2010). Chem. Mater. 22, 3283-3289.

Han, H., Park, J., Nam, S. Y., Kim, K. J., Choi, G. M., Parkin, S. S. P., Jang, H. M. \& Irvine, J. T. S. (2019). Nat. Commun. 10, 1471.

Hwang, J., Rao, R. R., Giordano, L., Katayama, Y., Yu, Y. \& ShaoHorn, Y. (2017). Science, 358, 751-756.

ICDD (2018). PDF-4+ 2019. International Centre for Diffraction Data, Newtown Square, PA, USA.

Jiang, Y., Geng, Z., Sun, Y., Wang, X., Huang, K., Cong, Y., Shi, F., Wang, Y., Zhang, W. \& Feng, S. (2020). ACS Sustain. Chem. Eng. 8, 302-310.

Karsai, F., Tran, F. \& Blaha, P. (2017). Comput. Phys. Commun. 220, 230-238.

Katz, M. B., Graham, G. W., Duan, Y. W., Liu, H., Adamo, C., Schlom, D. G. \& Pan, X. Q. (2011). J. Am. Chem. Soc. 133, 18090-18093.

Katz, M. B., Zhang, S. Y., Duan, Y. W., Wang, H. J., Fang, M. H., Zhang, K., Li, B. H., Graham, G. W. \& Pan, X. Q. (2012). J. Catal. 293, 145-148.

Keav, S., Matam, S. K., Ferri, D. \& Weidenkaff, A. (2014). Catalysts, 4, 226-255.

Kim, D., Bliem, R., Hess, F., Gallet, J. J. \& Yildiz, B. (2020). J. Am. Chem. Soc. 142, 3548-3563.

Kobsiriphat, W., Madsen, B. D., Wang, Y., Shah, M., Marks, L. D. \& Barnett, S. A. (2010). J. Electrochem. Soc. 157, B279-B284.

Koo, B., Kim, K., Kim, J. K., Kwon, H., Han, J. W. \& Jung, W. (2018). Joule, 2, 1476-1499. 
Kraushofer, F., Jakub, Z., Bichler, M., Hulva, J., Drmota, P., Weinold, M., Schmid, M., Setvin, M., Diebold, U., Blaha, P. \& Parkinson, G. S. (2018). J. Phys. Chem. C, 122, 1657-1669.

Kuhn, M., Hashimoto, S., Sato, K., Yashiro, K. \& Mizusaki, J. (2011). Solid State Ionics, 195, 7-15.

Kwon, O., Sengodan, S., Kim, K., Kim, G., Jeong, H. Y., Shin, J., Ju, Y. W. \& Han, J. W. (2017). Nat. Commun. 8, 15967.

Lee, J. G., Myung, J. H., Naden, A. B., Jeon, O. S., Shul, Y. G. \& Irvine, J. T. S. (2020). Adv. Energy Mater. 10, 1903693.

LeValley, T. L., Richard, A. R. \& Fan, M. H. (2014). Int. J. Hydrogen Energy, 39, 16983-17000.

Lim, H. S., Lee, M., Kang, D. \& Lee, J. W. (2018). Int. J. Hydrogen Energy, 43, 20580-20590.

Lindenthal, L., Rameshan, R., Summerer, H., Ruh, T., Popovic, J., Nenning, A., Löffler, S., Opitz, A. K., Blaha, P. \& Rameshan, C. (2020). Catalysts, 10, 268.

Lu, L. Y., Ni, C. S., Cassidy, M. \& Irvine, J. T. S. (2016). J. Mater. Chem. A, 4, 11708-11718.

Monkhorst, H. J. \& Pack, J. D. (1976). Phys. Rev. B, 13, 5188-5192.

Neagu, D., Oh, T. S., Miller, D. N., Menard, H., Bukhari, S. M., Gamble, S. R., Gorte, R. J., Vohs, J. M. \& Irvine, J. T. S. (2015). Nat. Commun. 6, 8120 .

Neagu, D., Tsekouras, G., Miller, D. N., Ménard, H. \& Irvine, J. T. S. (2013). Nat. Chem. 5, 916-923.

Nilsson, F., Sakuma, R. \& Aryasetiawan, F. (2013). Phys. Rev. B, 88, 125123.

Nishihata, Y., Mizuki, J., Akao, T., Tanaka, H., Uenishi, M., Kimura, M., Okamoto, T. \& Hamada, N. (2002). Nature, 418, 164-167.

Oh, T. S., Rahani, E. K., Neagu, D., Irvine, J. T. S., Shenoy, V. B., Gorte, R. J. \& Vohs, J. M. (2015). J. Phys. Chem. Lett. 6, 5106-5110.

Opitz, A. K., Nenning, A., Rameshan, C., Kubicek, M., Götsch, T., Blume, R., Hävecker, M., Knop-Gericke, A., Rupprechter, G., Klötzer, B. \& Fleig, J. (2017). Appl. Mater. Interfaces, 9, 3584735860.

Opitz, A. K., Nenning, A., Rameshan, C., Rameshan, R., Blume, R., Hävecker, M., Knop-Gericke, A., Rupprechter, G., Fleig, J. \& Klötzer, B. (2015). Angew. Chem. Int. Ed. 54, 2628-2632.

Opitz, A. K., Rameshan, C., Kubicek, M., Rupp, G. M., Nenning, A., Götsch, T., Blume, R., Hävecker, M., Knop-Gericke, A., Rupprechter, G., Klötzer, B. \& Fleig, J. (2018). Top. Catal. 61, 2129-2141.

Pechini, M. P. (1967). US Patent 3330697. Sprague Electric Company.

Perdew, J. P., Burke, K. \& Ernzerhof, M. (1996). Phys. Rev. Lett. 77, 3865-3868.
Popovic, J., Lindenthal, L., Rameshan, R., Ruh, T., Nenning, A., Löffler, S., Opitz, A. K. \& Rameshan, C. (2020). Catalysts, 10, 582.

Preis, W., Bucher, E. \& Sitte, W. (2004). Solid State Ionics, 175, 393 397.

Rahimi, F., Jafari, A. K., Hsu, C. A., Ferekides, C. S. \& Hoff, A. M. (2019). Org. Electron. 75, 105397.

Rousseau, S., Marie, O., Bazin, P., Daturi, M., Verdier, S. \& Harlé, V. (2010). J. Am. Chem. Soc. 132, 10832-10841.

Schmid, A., Rupp, G. M. \& Fleig, J. (2018a). Chem. Mater. 30, $4242-$ 4252.

Schmid, A., Rupp, G. M. \& Fleig, J. (2018b). Phys. Chem. Chem. Phys. 20, 12016-12026.

Sławiński, W., Przeniosło, R., Sosnowska, I. \& Suard, E. (2005). J. Phys. Condens. Matter, 17, 4605-4614.

Søgaard, M., Vang Hendriksen, P. \& Mogensen, M. (2007). J. Solid State Chem. 180, 1489-1503.

Steiger, P., Alxneit, I. \& Ferri, D. (2019). Acta Mater. 164, 568-576.

Streltsov, V. A. \& Ishizawa, N. (1999). Acta Cryst. B55, 1-7.

Sun, Y. F., Li, J. H., Zeng, Y. M., Amirkhiz, B. S., Wang, M. N., Behnamian, Y. \& Luo, J. L. (2015). J. Mater. Chem. A, 3, 1104811056.

Tan, L., Gu, X. H., Yang, L., Zhang, L. X., Wang, C. Q. \& Xu, N. P. (2003). Sep. Purif. Technol. 32, 307-312.

Tanaka, H., Uenishi, M., Taniguchi, M., Tan, I., Narita, K., Kimura, M., Kaneko, K., Nishihata, Y. \& Mizuki, J. (2006). Catal. Today, 117, 321-328.

Thalinger, R., Gocyla, M., Heggen, M., Klötzer, B. \& Penner, S. (2015). J. Phys. Chem. C, 119, 22050-22056.

Toby, B. H. (2006). Powder Diffr. 21, 67-70.

Vieten, J., Bulfin, B., Huck, P., Horton, M., Guban, D., Zhu, L. Y., Lu, Y. J., Persson, K. A., Roeb, M. \& Sattler, C. (2019). Energy Environ. Sci. 12, 1369-1384.

Wang, H. C., Bao, Z., Tsai, H. Y., Tang, A. C. \& Liu, R. S. (2018). Small, 14, 1702433.

Wang, Y., Wang, Y., Ren, W., Liu, P., Zhao, H., Chen, J., Deng, J. \& Xing, X. (2015). Phys. Chem. Chem. Phys. 17, 29097-29102.

Wollan, E. O. \& Koehler, W. C. (1955). Phys. Rev. 100, 545-563.

Yates, J. T. \& Campbell, C. T. (2011). Proc. Natl Acad. Sci. USA, 108, 911-916.

Yoon, K. J., Zink, P. A., Gopalan, S., Pal, U. B. \& Pederson, L. R. (2009). J. Electrochem. Soc. 156, B795-B800.

Zeng, Z. C., Xu, Y. S., Zhang, Z. S., Gao, Z. S., Luo, M., Yin, Z. Y., Zhang, C., Xu, J., Huang, B. L., Luo, F., Du, Y. P. \& Yan, C. H. (2020). Chem. Soc. Rev. 49, 1109-1143. 